九州大学学術情報リポジトリ

Kyushu University Institutional Repository

\title{
An investigation of wall-anisotropy expressions and length-scale equations for non-linear eddy- viscosity models
}

$A B E$, Ken-ichi

Department of Aeronautics and Astronautics, Faculty of Engineering, Kyushu University :

Professor

Jang, Y. - J.

Department of Aeronautics, Imperial College of Science, Technology and Medicine

Leschziner, M. A.

Department of Aeronautics, Imperial College of Science, Technology and Medicine

http://hdl. handle. net/2324/4150688

出版情報: International Journal of Heat and Fluid Flow. 24 (2)，pp.181-198，2003-04. Elsevier バージョン：

権利関係 : 


\title{
An investigation of wall-anisotropy expressions and length-scale equations for non-linear eddy-viscosity models
}

\author{
K. Abe ${ }^{1}$, Y.-J. Jang ${ }^{2}$ and M. A. Leschziner ${ }^{2}$ \\ ${ }^{1}$ Department of Aeronautics and Astronautics, Kyushu University, Fukuoka, Japan \\ ${ }^{2}$ Department of Aeronautics, Imperial College of Science, Technology and Medicine, \\ London, UK
}

(Corresponding author: Dr. Ken-ichi Abe, Department of Aeronautics and Astronautics, Kyushu University, Hakozaki, Higashi-ku, Fukuoka 812-8581, Japan.)

\begin{abstract}
New closure approximations are proposed, within the framework of non-linear eddy-viscosity modeling, which aim specifically at an improved representation of near-wall anisotropy in both shear and stagnation flows. The main novel element is the introduction of tensorial terms, alongside strain and vorticity, which depend on wall-direction indicators and which procure the correct asymptotic near-wall behavior of the Reynolds stresses. The newly formulated non-linear constitutive equation for the Reynolds stresses is combined with low-Reynoldsnumber forms of equations for the rate of dissipation $\varepsilon$ or the specific dissipation $\omega$, the latter incorporating a number of new features into the established form of the equation. The predictive performance of three model variants is investigated by reference to three test flows: a plane channel flow, a separated flow in a channel with periodic hill-shaped obstacles on one wall and a plane-impinging jet. It is shown that the new model elements result in a substantially improved representation of the Reynolds-stress field at the wall, especially in the wall-normal Reynolds stress. One of the variants includes the use of the modified $\omega$-equation, and it is shown that this model performs especially well in the presence of separation.
\end{abstract}

\section{Keywords}

Non-linear Eddy-Viscosity Model, Reynolds Stress Tensor, Near-Wall Anisotropy Length-Scale Equation, Separated Flow, Impinging Flow 


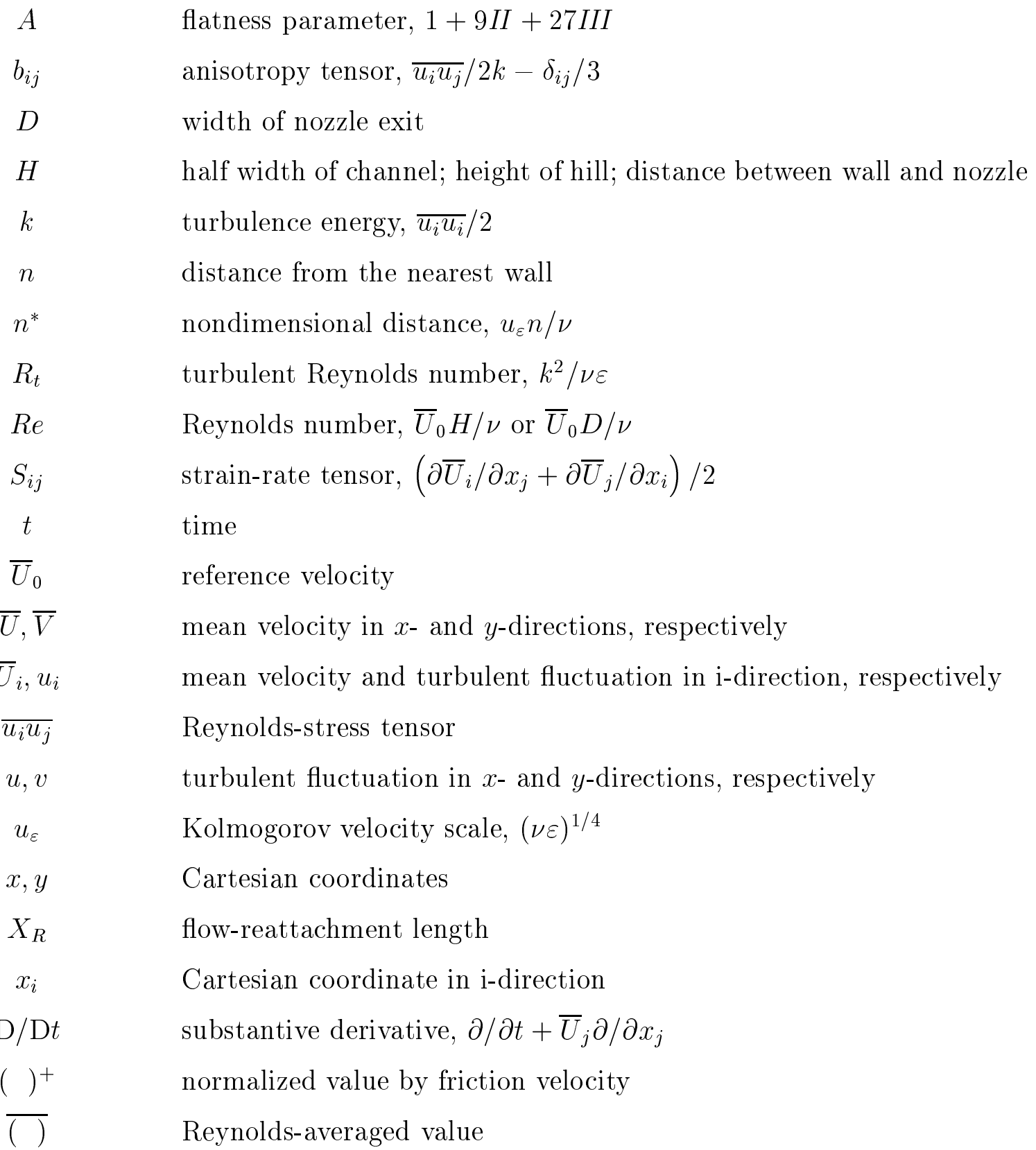

Greek symbols

$\begin{array}{cl}\delta_{i j} & \text { Kronecker delta } \\ \varepsilon & \text { dissipation rate of turbulence energy, } \nu \overline{\left(\partial u_{i} / \partial x_{j}\right)\left(\partial u_{i} / \partial x_{j}\right)} \\ \nu, \nu_{t} & \text { kinematic and eddy viscosity, respectively } \\ \tau, \tau_{d} & \text { characteristic time scales for turbulence } \\ \Omega_{i j} & \text { vorticity tensor, }\left(\partial \bar{U}_{i} / \partial x_{j}-\partial \bar{U}_{j} / \partial x_{i}\right) / 2 \\ \omega & \text { specific dissipation }\end{array}$


second anisotropy invariant, $-\frac{1}{2} b_{i j} b_{j i}$

third anisotropy invariant, $\frac{1}{3} b_{i j} b_{j k} b_{k i}$ 


\section{Introduction}

Reynolds-averaged turbulence models continue to constitute the principal approach for representing the effects of turbulence in prediction procedures for engineering flows. Among them, algebraic relations that link the Reynolds-stress components to mean velocity gradients have been widely used. A great deal of effort has gone, especially over the past decade, into the development and improvement of constitutive expressions that relate the Reynolds-stress tensor, non-linearly, to the strain-rate and vorticity tensors. Examples are the non-linear eddy-viscosity models (NLEVMs) of Suga (1995), Craft et al (1996), Apsley and Leschziner (1998), Suga and Abe (2000) and Wallin and Johansson (2000), the last arising from a formal inversion of an algebraic approximation of a second-moment-transport closure to give explicit algebraic relations for the Reynolds stresses. Such models are designed to capture anisotropy and to represent, realistically, the response of turbulence to curvature and normal straining, without the elaboration imposed by full second-moment closure.

One issue that has been the subject of much attention, in all categories of models, is the wall-limiting behavior returned by low-Reynolds-number (LRN) forms that are designed to be applicable across the viscous sublayer, down to the wall. This is, naturally, of particular concern in relation to heat and mass transfer at walls, in which case the turbulence property of the near-wall layer plays a critical role. In the context of linear eddy-viscosity models (LEVMs), key aspects of the near-wall behavior are the decay of turbulence energy, turbulent viscosity and the turbulent shear stress, as well as the behavior of the dissipation rate or other

length-scale surrogates. Numerous LEVMs have been proposed in the 1980s and 1990s, each distinguished from others, principally, by particular forms of viscosity-related damping functions intended to control the wall-limiting behavior.

In the case of non-linear models, securing the correct representation of the wall-limiting behavior is considerably more challenging than in linear forms. Here, the requirement is for the model to correctly represent the variation of all stress components as the wall is approached, and this needs to be done in the face of especially high levels of anisotropy and rapid variations thereof. Inevitably, this entails, again, the use of viscosity-dependent damping functions, but must also involve the introduction of carefully designed functions of strain and vorticity invariants. There remains much room for improving current NLEVMs in this respect. Such improvements are imperative if NLEVMs are to form a credible basis for 
advanced scalar-flux models (e.g., Abe and Suga (2001a, 2001b)) which lean heavily on the correct prediction of all Reynolds-stress components, especially in the case of complex flows involving separation and impingement where all flux components affect the mean scalar field.

The present paper is a contribution to the ongoing search for better non-linear eddyviscosity models for complex flows. The emphasis of the contribution is on improved forms of the stress-strain/vorticity relations that are designed to procure the correct stress anisotropy at the wall. In addition, the paper examines the influence of representing the length scale, alternatively, via transport equations for the dissipation rate, $\varepsilon$, and the specific dissipation, $\omega=\varepsilon / k$. The characteristics of the proposed models are illustrated by application of the models to three flows: a plane channel flow, a separated flow in a channel with wavy-wall-like periodic constrictions and a plane impinging jet.

\section{Stress-strain Relation for Reynolds Stress Tensor}

\subsection{Basic formulation}

The key objective pursued below is to provide a framework for evaluating the Reynolds-stress tensor $\overline{u_{i} u_{j}}$. The starting point is the model of Abe et al. (1997).

In what follows, use is made of the anisotropy tensor $b_{i j}$, the strain-rate tensor $S_{i j}$ and the vorticity tensor $\Omega_{i j}$, the last two normalized with the turbulent time scale:

$$
\begin{gathered}
b_{i j}=\frac{\overline{u_{i} u_{j}}}{2 k}-\frac{\delta_{i j}}{3}, \quad S_{i j}=\frac{1}{2}\left(\frac{\partial \bar{U}_{i}}{\partial x_{j}}+\frac{\partial \bar{U}_{j}}{\partial x_{i}}\right), \quad \Omega_{i j}=\frac{1}{2}\left(\frac{\partial \bar{U}_{i}}{\partial x_{j}}-\frac{\partial \bar{U}_{j}}{\partial x_{i}}\right) \\
b_{i j}^{*}=C_{D} b_{i j}, \quad S_{i j}^{*}=C_{D} \tau S_{i j}, \quad \Omega_{i j}^{*}=C_{D} \tau \Omega_{i j},
\end{gathered}
$$

where

$$
\tau=\frac{\nu_{t}}{k}, \quad \nu_{t}=C_{\mu} f_{\mu} \frac{k^{2}}{\varepsilon}, \quad C_{D}=0.8, \quad C_{\mu}=0.12 .
$$

To represent the damping effects of viscosity, use is made of Abe et al's damping function:

$$
f_{\mu}=\left[1+\frac{35}{R_{t}^{\frac{3}{4}}} \exp \left\{-\left(\frac{R_{t}}{30}\right)^{\frac{3}{4}}\right\}\right]\left\{1-f_{w}(26)\right\}
$$

where

$$
f_{w}(\xi)=\exp \left\{-\left(\frac{n^{*}}{\xi}\right)^{2}\right\} .
$$

In Eqs. (4) and (5), $R_{t}\left(=k^{2} / \nu \varepsilon\right)$ is the turbulent Reynolds number, $n^{*}\left(=u_{\varepsilon} n / \nu\right)$ is the non-dimensional wall distance and $\xi$ is a prescribed constant. 
The basic constitutive relation for the anisotropy tensor is that proposed by Abe et al. (1997),

$$
b_{i j}^{*}=\frac{1}{1+\frac{22}{3} \Omega^{* 2}+\frac{2}{3}\left(\Omega^{*^{2}}-S^{* 2}\right) f_{B}}\left\{-S_{i j}^{*}-2\left(S_{i k}^{*} \Omega_{k j}^{*}-\Omega_{i k}^{*} S_{k j}^{*}\right)+2\left(S_{i k}^{*} S_{k j}^{*}-\frac{\delta_{i j}}{3} S^{* 2}\right)\right\},
$$

where

$$
\begin{gathered}
f_{B}=1+C_{\eta}\left(\Omega^{*}-S^{*}\right) \\
S^{* 2}=S_{m n}^{*} S_{m n}^{*}, \quad \Omega^{* 2}=\Omega_{m n}^{*} \Omega_{m n}^{*}, \quad S^{*}=\sqrt{S^{* 2}}, \quad \Omega^{*}=\sqrt{\Omega^{* 2}}, \quad C_{\eta}=100 .
\end{gathered}
$$

It is noted here, however, that the function $f_{B}$ is not identical to the original form, but has been modified slightly in connection with further modifications described below. The arguments leading to Eq. (6) are described in Abe et al. (1997).

To facilitate further considerations, relation (6) is decomposed into its linear and nonlinear parts:

$$
b_{i j}^{*}={ }^{1} b_{i j}^{*}+{ }^{2} b_{i j}^{*}
$$

where

$$
\begin{aligned}
& { }^{1} b_{i j}^{*}=-C_{B} S_{i j}^{*} \\
& { }^{2} b_{i j}^{*}=C_{B}\left\{-2\left(S_{i k}^{*} \Omega_{k j}^{*}-\Omega_{i k}^{*} S_{k j}^{*}\right)+2\left(S_{i k}^{*} S_{k j}^{*}-\frac{\delta_{i j}}{3} S^{* 2}\right)\right\} .
\end{aligned}
$$

In Eq. (9), the coefficient $C_{B}$ arises directly from Eq. (6) as

$$
C_{B}=\frac{1}{1+\frac{22}{3} \Omega^{*^{2}}+\frac{2}{3}\left(\Omega^{*^{2}}-S^{*^{2}}\right) f_{B}}
$$

Modifications to follow relate to the non-linear group in Eq. (8).

\subsection{Modification for strong normal strain}

Although the basic formulation Eq. (6) is designed to give reasonable predictions for both wall and homogeneous shear flows (Abe et al. (1997)), it does not perform well in flows in which normal straining is dominant - for example, in plain strain, axisymmetric contraction and axisymmetric expansion. This weakness is addressed by the introduction of the new model component, 


$$
{ }^{s} b_{i j}^{*}=-C_{B} f_{s 1} S_{i j}^{*}+2 C_{B} f_{s 2}\left(S_{i k}^{*} S_{k j}^{*}-\frac{\delta_{i j}}{3} S^{* 2}\right)
$$

where

$$
\begin{gathered}
f_{s 1}=f_{r 1} f_{r 2} C_{s 1}\left(\Omega^{* 2}-S^{* 2}\right), \quad f_{s 2}=-f_{r 1} f_{r 2}\left\{1+C_{s 2}\left(\Omega^{*}-S^{*}\right)\right\} \\
f_{r 1}=\frac{\Omega^{2}-S^{2}}{\Omega^{2}+S^{2}}, \quad f_{r 2}=\frac{S^{2}}{\Omega^{2}+S^{2}}, \\
S^{2}=S_{m n} S_{m n}, \quad \Omega^{2}=\Omega_{m n} \Omega_{m n}, \quad C_{s 1}=0.15 C_{\eta}, \quad C_{s 2}=0.07 C_{\eta} .
\end{gathered}
$$

Thus, with the above modification included,

$$
b_{i j}^{*}={ }^{1} b_{i j}^{*}+{ }^{2} b_{i j}^{*}+{ }^{s} b_{i j}^{*}
$$

Fragment Eq. (11), when added to Eq. (8), allows the model to return a reasonable representation for strong normal straining, and it also guarantees realizability. The function $f_{r 1}$ ensures that relation (11) has no effect in pure shear, while the function $f_{r 2}$ counteracts effects arising from Eq. (11) in the presence of strong system rotation, ensuring realizability under the condition $\Omega^{2} \gg S^{2}$. All model functions and coefficients in Eq. (11) have been calibrated by reference to baseline flows described below.

The performance of the modified model in homogenous-flow conditions is illustrated in Fig. 1. The figure shows the response of the anisotropy components, evaluated from Eq. (2), to pure shear strain, plain strain, axisymmetric contraction and axisymmetric expansion. As seen from Fig. 1 (a), reasonable shear-stress values arise for both near-wall shear $\left[\frac{k}{\varepsilon} \sqrt{2 S^{2}} \sim\right.$

3.3] and homogeneous shear $\left[\frac{k}{\varepsilon} \sqrt{2 S^{2}} \sim 6\right]$, with the realizability condition secured for strong strain (Abe et al. (1997)). As for normal straining, plots (b)-(d) in Fig. 1 demonstrate that the normal-stress separation is realistic and that realizability is maintained even at high strain rates.

\subsection{Modification for strong anisotropy in near-wall region}

In common with other NLEVMs, the model (13) does not return an adequate representation of anisotropy close to the wall. To improve the predictive performance of Eq. (13) in this respect, the following additive fragment is proposed: 


$$
\begin{gathered}
{ }^{w} b_{i j}^{*}=C_{D} f_{w}(26){ }^{w} b_{i j} \\
{ }^{w} b_{i j}=-\alpha_{w} \frac{1}{2}\left(d_{i} d_{j}-\frac{\delta_{i j}}{3} d_{k} d_{k}\right)+\left(1-f_{r 1}{ }^{2}\right)\{ \\
-\frac{\beta_{w} C_{w}}{1+C_{w} \sqrt{S^{* * 2} \Omega^{* * 2}}}\left(S_{i k}^{* *} \Omega_{k j}^{* *}-\Omega_{i k}^{* *} S_{k j}^{* *}\right) \\
\left.\quad+\frac{\gamma_{w} C_{w}}{1+C_{w} S^{* * 2}}\left(S_{i k}^{* *} S_{k j}^{* *}-\frac{\delta_{i j}}{3} S^{* * 2}\right)\right\}
\end{gathered}
$$

where

$$
S_{i j}^{* *}=\tau_{d} S_{i j}, \quad \Omega_{i j}^{* *}=\tau_{d} \Omega_{i j}, \quad S^{* * 2}=S_{m n}^{* *} S_{m n}^{* *}, \quad \Omega^{* * 2}=\Omega_{m n}^{* *} \Omega_{m n}^{* *} .
$$

In Eq. (15), $d_{i}$ is a unit vector representing the wall-normal direction. In this study, $d_{i}$ takes the form,

$$
d_{i}=\frac{N_{i}}{\sqrt{N_{k} N_{k}}}, \quad N_{i}=\frac{\partial l_{d}}{\partial x_{i}}, \quad l_{d}=n .
$$

Although the wall distance $(n)$ is used above, alternative variables could be proposed, as long as its spatial gradient vector represents realistically the wall-normal direction. Possibilities include $k^{3 / 2} / \varepsilon$ and a combination of this group with anisotropy parameters which show characteristic wall-normal variations (e.g., Jakirlic and Hanjalic (1995); Craft and Launder (1996); Iacovides and Raisee (1997)).

The time scale $\tau_{d}$ is required to be associated with the near-wall turbulence and must therefore be expected to be related not only to the turbulent scales but also to the viscosity. Hence, a general functional relationship is of the form:

$$
\tau_{d}=F\left(\frac{k}{\varepsilon}, \sqrt{\frac{\nu}{\varepsilon}}\right)
$$

Following Durbin (1993), a model that may be adopted for Eq. (18) is

$$
\tau_{d}=\max \left(\frac{k}{\varepsilon}, 6 \sqrt{\frac{\nu}{\varepsilon}}\right)
$$

In this study, however, a blending of the two time scales, with weighting controlled by $f_{w}$, has been found to give better performance. Thus, the time scale is approximated by:

$$
\tau_{d}=\left\{1-f_{w}(15)\right\} \frac{k}{\varepsilon}+f_{w}(15) \sqrt{\frac{\nu}{\varepsilon}} .
$$

This expression provides a continuous variation of the time scale with the same limiting levels as Durbin's model and with equal weight given to the time scales in the center of the buffer layer. 
In computations to follow, we examine two sets of model coefficients:

$$
\begin{array}{lll}
\text { Model 1: } & \alpha_{w}=1, \quad \beta_{w}=\frac{1}{4}, \quad \gamma_{w}=1.5, \quad C_{w}=0.5 \\
\text { Model 2: } & \alpha_{w}=0, \quad \beta_{w}=\frac{5}{12}, \quad \gamma_{w}=0.5, \quad C_{w}=1.0
\end{array}
$$

Model 2 is evidently based on the concept that the near-wall anisotropy is controlled only by the strength of the strain-rate components (Suga (1995), Apsley and Leschziner (1998), Wallin and Johansson (2000)). Although this concept has been widely used, it causes a misrepresentation of the anisotropy at stagnation, separation and reattachment points where the strain rate vanishes. Without any correction, an isotropic state is returned. In reality, however, the near-wall flow is highly anisotropic, and while the wall-asymptotic variation of the individual anisotropy components depends on the nature of the flow above the immediate near-wall layer, typical variations are of the form $b_{22} \rightarrow-1 / 3, b_{11} \rightarrow O(0.4)$ and $b_{33} \rightarrow$ $O(-0.07)$, with the first being unconditionally correct. The consequence of $b_{22} \rightarrow-1 / 3$ is that turbulence approaches the two-component line $A=1+9 I I+27 I I I=0$, where $A$ is Lumley's flatness parameter, $I I\left(=-\frac{1}{2} b_{i j} b_{j i}\right)$ and $I I I\left(=\frac{1}{3} b_{i j} b_{j k} b_{k i}\right)$ are respectively the second and the third invariants of the Reynolds-stress tensor. However, the manner in which this line is approached is not universal and depends on the nature and history of the flow bordering the near-wall region.

To some degree, adherence to the two-component limit can be secured by calibrating model coefficients by reference to DNS data for selected near-wall flows, as has been done by Apsley and Leschziner (1998). However, this is not a general approach and does not result in the correct variations for any significant range of conditions.

Model 1 aims to procure the correct asymptotic approach to two-component turbulence for a range of flow conditions. Its wall-related fragments reflect the notion that near-wall anisotropy should be controlled preferentially by wall-proximity terms, Eq. (15). Among them, the first term in Eq. (15) affects the anisotropy by damping the wall-normal stress and enhancing the two other components, regardless of the strain rate. As the flow is allowed to affect the approach to the two-component state, the expectation is that the model will be applicable to both shear conditions $(I I I>0)$ and axisymmetric contraction $(I I I<0)$.

The general form of the constitutive equation for the anisotropy used in calculations to 
follow is:

$$
b_{i j}^{*}={ }^{1} b_{i j}^{*}+\left\{1-f_{w}(26)\right\}\left\{{ }^{2} b_{i j}^{*}+{ }^{s} b_{i j}^{*}\right\}+{ }^{w} b_{i j}^{*},
$$

where ${ }^{1} b_{i j}^{*},{ }^{2} b_{i j}^{*},{ }^{s} b_{i j}^{*}$ and ${ }^{w} b_{i j}^{*}$ are given by Eqs. (9), (11) and (14), respectively. This form concurs with Eq. (13) in the case of homogeneous flows that are free from wall-related influences. The model (22) nullifies the non-linear and normal-strain fragments very close to the wall, where the anisotropy is controlled by Eqs. (14) and (15). The Reynolds stresses may be evaluated from Eqs. (2) and (22) as follows:

$$
\overline{u_{i} u_{j}}=\frac{2}{3} k \delta_{i j}+2 k b_{i j}=\frac{2}{3} k \delta_{i j}+2 k\left(\frac{b_{i j}^{*}}{C_{D}}\right) .
$$

Attention is drawn to the fact that the model (14) is independent of the non-linear model fragments in Eq. (9). Hence, it can be employed, in principle, in conjunction with any other model using an algebraic stress-strain relationship.

\section{Turbulence-Transport Equations}

\subsection{Turbulence-energy equation}

The turbulence energy, required for the time scale and turbulent viscosity in Eq. (3), is determined from the usual form of the transport equation:

$$
\frac{\mathrm{D} k}{\mathrm{D} t}=\frac{\partial}{\partial x_{j}}\left\{\left(\nu+\frac{\nu_{t}}{\sigma_{k}}\right) \frac{\partial k}{\partial x_{j}}\right\}-\overline{u_{i} u_{j}} \frac{\partial \bar{U}_{i}}{\partial x_{j}}-\varepsilon,
$$

where

$$
\sigma_{k}=\frac{1.2}{f_{t}}, \quad f_{t}=1+5.0 f_{w}(5)
$$

As regards the turbulent diffusion, the value of $\sigma_{k}$ must be carefully calibrated because the present model adopts a value of $C_{\mu}$ different from the usual 0.09 , in conjunction with $C_{B}$ of Eq. (9). Also, it has been demonstrated by DNS data (Kim et al. (1990)) that the turbulent diffusion in the $k$-equation becomes larger as the wall is approached, and thus a constant value of $\sigma_{k}$ is inapplicable to the whole flow region (Nagano and Shimada (1995)). After careful calibrations, as part of this study, $\sigma_{k}=1.2$ was adopted, together with a model function $f_{t}$ given by Eq. (25). This function increases the turbulent diffusion in the vicinity of the wall and, as a result, the turbulent energy budget can be successfully predicted, as shown in Section 5.1. 


\subsection{Length-scale equations}

Two alternative length-scale-governing equations are investigated, one for the dissipation-rate $\varepsilon$ and the other for the specific dissipation $\omega=\varepsilon / k$.

The dissipation-rate equation is that proposed by Abe et al. (1997) with minor modifications:

$$
\frac{\mathrm{D} \varepsilon}{\mathrm{D} t}=\frac{\partial}{\partial x_{j}}\left\{\left(\nu+\frac{\nu_{t}}{\sigma_{\varepsilon}}\right) \frac{\partial \varepsilon}{\partial x_{j}}\right\}-C_{\varepsilon 1} \frac{\varepsilon}{k} \overline{u_{i} u_{j}} \frac{\partial \bar{U}_{i}}{\partial x_{j}}-C_{\varepsilon 2} f_{\varepsilon} \frac{\varepsilon^{2}}{k},
$$

where

$$
\begin{gathered}
f_{\varepsilon}=\left[1-0.3 \exp \left\{-\left(\frac{R_{t}}{6.5}\right)^{2}\right\}\right]\left\{1-f_{w}(3.3)\right\} \\
C_{\varepsilon 1}=1.45, \quad C_{\varepsilon 2}=1.83, \quad \sigma_{\varepsilon}=\frac{1.5}{f_{t}} .
\end{gathered}
$$

The constant $C_{\varepsilon 2}$ is lower than the standard value 1.92. This choice is rooted in modeling considerations by Gatski and Speziale (1993) and Apsley and Leschziner (1998) which pertain to the use of the $\omega$ equation. These suggest that the favorable properties of the $\omega$-equation in boundary layers subjected to adverse-pressure gradient are due, in part, to a value of the coefficient $\beta$ (see below) being lower than that implied by the standard value of the equivalent coefficient $C_{\varepsilon 2}$. It is noted that the value of $\sigma_{\varepsilon}$ needs to be calibrated carefully for the same reasons as those discussed earlier in relation to the $k$-equation. In this study, $\sigma_{\varepsilon}=1.5$ and the function $f_{t}$ in Eq. (25) have been adopted to successfully reproduce the log-low profile and the correct behavior of the dissipation rate in plane-channel flow.

The $\omega$-equation used here is as follows:

$$
\begin{gathered}
\omega=\frac{\varepsilon}{\beta^{*} k} \\
\frac{\mathrm{D} \omega}{\mathrm{D} t}=\frac{\partial}{\partial x_{j}}\left\{\left(\nu+\frac{\nu_{t}}{\sigma_{\omega}}\right) \frac{\partial \omega}{\partial x_{j}}\right\}-\alpha \frac{\omega}{k} \overline{u_{i} u_{j}} \frac{\partial \bar{U}_{i}}{\partial x_{j}}-\beta \omega^{2}+E_{\omega},
\end{gathered}
$$

where

$$
\begin{gathered}
E_{\omega}=\left\{1-f_{w}(600)\right\} C_{\omega 1} \tau \frac{\partial k}{\partial x_{j}} \frac{\partial \omega}{\partial x_{j}} \\
\beta^{*}=\beta_{0} \frac{\left(\frac{\beta}{3 \beta_{0}}\right)+\left(\frac{R_{t w}}{9}\right)^{4}}{1+\left(\frac{R_{t w}}{9}\right)^{4}}, \quad R_{t w}=\frac{k}{\nu \omega}, \quad \sigma_{\omega}=\frac{1.5}{f_{t}} \\
\beta_{0}=0.09, \quad \alpha=C_{\varepsilon 1}-1=0.45, \quad \beta=\beta_{0}\left(C_{\varepsilon 2}-1\right)=0.0747, \quad C_{\omega 1}=1.5 .
\end{gathered}
$$

In Eq. (31), $\tau$ is as defined by Eq. (3). While this form is based on that of Wilcox (1994), it includes a number of influential modifications. 
First, the mixed-derivative term $E_{\omega}$, Eq. (31), is introduced. A mixed-derivative term, though not identical to $E_{\omega}$, arises naturally in the transformation of the $\varepsilon$-equation to the $\omega$ form, but is omitted from Wilcox's model. This term plays an important role (Menter (1994)) in securing the correct behavior of the equation in the region in which a shear layer borders a free stream. The coefficients in Eq. (31) have been calibrated by reference to the channel-flow computations discussed in Section 5.1.

Second, the coefficient $\alpha$ is lower than Wilcox's value of $5 / 9$. This value implies $C_{\varepsilon 1}=$ 1.56, which is much larger than the generally accepted value of 1.44 . This difference is a further reason for the fact that Wilcox's $\omega$-equation tends to give longer recirculation zones than the $\varepsilon$-equation. While this elongation gives better accord with experimental data for backward-facing-step flow, there are examples in which the model returns excessively long recirculation bubbles for other cases - for example, in computations contributed to the 9 th IAHR-ERCOFTAC Workshop on Refined Turbulence Modeling, Jakirlic et al. (2001), for a channel flow constricted by periodic 'hills' (Temmerman and Leschziner (2001)). This is despite the fact that the model takes no account of the effects of anisotropy and streamline curvature, which are known to damp turbulence and thus inhibit reattachment.

Based on the above observations, the values adopted here for the constants $\alpha$ and $\beta$ are those derived from the $\varepsilon$-equation (26). The coefficient $\sigma_{\omega}$ is similarly taken over from the $\varepsilon$-equation. The resulting much closer compatibility between the present $\omega$ - and $\varepsilon$-equations, relative to Wilcox's form, may be argued to permit a fairer assessment to be made of the relative merits of using either $\varepsilon$ or $\omega$ as a basis for determining the length scale. When the latter route is used, Eq. (29) provides the means of extracting $\varepsilon$ from $\omega$, which is required in various other model components and equations (e.g., Eqs. (3), (19) and (24)).

The predictive performance of three model variants is investigated in computations presented in Section 4. The first variant, designated Model 1, combines formulation (20), with the $\varepsilon$-equation (26). Model 2 combines the formulation (21) with the same $\varepsilon$-equation. The third variant, designated Model 3, combines Eq. (20) with the w-equation (30). Comparisons between Model 1 and Model 2 are intended to provide information on the role and effectiveness of the wall-related anisotropy fragments, while comparisons between Model 1 and Model 3 are expected to highlight the consequence of alternative choices of surrogate length-scale variables. 


\section{Test Cases and Computational Conditions}

Three flow configurations have been selected to illustrate the properties of the models formulated in Sections 2 and 3. The first is a fully-developed plane-channel flow at $R e=6875$, based on mean-flow velocity and half-channel height, for which DNS data have been reported by Kim et al. (1990). The second case (referred to a 'periodic-hill flow') is a separated, spanwise-homogeneous flow in a periodically constricted channel, with the constrictions being hill-shaped and 9 hill-height apart. The Reynolds number is 21200, based on mean velocity and channel height. For this geometry, highly-resolved LES data have been reported by Temmerman and Leschziner (2001) and Temmerman et al. (2002). The third flow is a plane impinging jet at $R e=6000$, based on maximum jet-exit velocity and nozzle width, for which LES data have been reported by Abe and Suga (2001b).

The channel flow was used to examine some basic characteristics of the models, specifically in relation to their response to near-wall shear. Computations were performed with 84 wallnormal nodes covering the full width of the channel. Streamwise homogeneity was imposed, yielding a 1D computational problem, with the mass-flow rate fixed in the computation.

The periodic-hill flow is shown in Fig. 2. The presence of massive separation between consecutive hills allows the models to be investigated for conditions representative of complex separated flows. The close-to-orthogonal wall-fitted computational grid consisted of $113 \times$ 65 nodes. Streamwise periodicity was imposed by an iterative coupling of inlet and outlet conditions of the segment shown in Fig. 2, with the mass-flow rate fixed in the computation.

The plane-impinging jet is shown in Fig. 3 and has been chosen to investigate the performance of the models in the presence of strong normal straining. A Cartesian grid of $115 \times$ 87 nodes was used to cover one half of the symmetric flow domain to a height of $y / D=8.77$. Conditions at the upper boundary were taken from the LES data of Abe and Suga (2001b), together with the assumption $R_{t}=300$ used to determine the rate of dissipation. At the outlet boundary, $x / D=13.7$, zero streamwise gradients were prescribed.

In all three cases above, no-slip conditions were specified at walls, and the wall-nearest

node was placed at $y^{+}<1$. Calculations were performed with the finite-volume procedure STREAM of Lien and Leschziner (1994a), followed by several improvements and substantially upgraded by Apsley and Leschziner (2000). This uses collocated storage on a non-orthogonal grid and approximates all variables with the aid of the UMIST scheme (Lien and Leschziner 
(1994b)), a TVD implementation of the QUICK scheme.

\section{Results and Discussion}

\subsection{Plane channel flow}

Figures $4-6$, each relating to one of the three models defined in Section 3, give results for the mean velocity, Reynolds stresses, budget of $k$, anisotropy components and anisotropy invariants, by comparison to DNS data. Note that the value of $\varepsilon$ for Model 3 is calculated from Eq. (29). For this particular flow, Model 1 and Model 2 return identical solutions for the mean velocity, budget of $k$ and shear-stress component, all being in good agreement with the DNS data. The agreement achieved with Model 3 for the mean velocity is slightly less good, suggesting a difference in the model-predicted wall-shear stress relative to the DNS value.

All three models return more than a fair representation of the normal stresses near the wall, with the models utilizing the wall-direction term in Eq. (15) doing best. The plots for anisotropy tensor $b_{i j}$ give an especially clear view of the ability of the models to resolve the intense near-wall anisotropy. Again, Model 1 and Model 3 perform especially well giving the correct wall-limiting value of $b_{22}=-1 / 3$. All three models wrongly predict a trend towards isotropy as the channel centerline is approached. This is a direct consequence of the vanishing velocity gradient at the channel center and is consistent with the state of isotropy predicted by Eq. (6) for $S^{2}=0$ in homogeneous shear, as seen from Fig. 1 (a).

The Reynolds-stress components may readily be used to obtain distributions for the invariants $I I, I I I$ and $A$. These are plotted in Figs. $4-6$ in the form of cross-channel profiles, as well as in the form of $I I / I I I$-loci in Lumley's realizability map. Consistent with earlier results, Models 1 and 3 do well, especially near the wall where both return a vanishing value for the flatness parameter. Both also represent well the extreme values of $I I$ and $I I I$ which identify the closest point to one-component turbulence. Both observations highlight, again, the important role played by the leading term in Eq. (15).

All three models are seen to satisfy realizability, but Model 1 does best in terms of representing both the stress field and the mean-flow velocity. Model 2, while generally satisfactory, shows some weaknesses very close to the wall. In particular, this model neither returns the correct limiting value $-1 / 3$ for $b_{22}$ nor gives the wall-limiting zero value for the flatness pa- 
rameter signifying two-component turbulence. While this defect may not be of consequence to the predicted mean flow in relatively simple shear flows, it will be shown below that it can have a detrimental effect on predictive accuracy in complex conditions.

\subsection{Periodic-hill flow}

Comparisons of model solutions for mean-flow properties and Reynolds-stress components with corresponding LES data of Temmerman and Leschziner (2001) are shown in Figs. 7 10. Figure 7 gives an overall view, in the form of model-predicted streamfunction plots (b)-(d), compared to the LES solution (a). The reattachment locations $X_{R} / H$ predicted by Models 1, 2 and 3 are, respectively, 4.23, 4.17 and 5.36, relative to the LES value 4.75. It is noted, by reference to the zero-streamline, that separation is predicted by Models 1 and 2 to occur too late, especially by Model 1, and that the reattachment behavior produced by these two models differs from those returned by the LES and Model 3. Specifically, the angle close to reattachment is steeper, indicating a faster recovery towards reattachment. While Model 3 over-estimates the length of the separation zone, it gives a more faithful representation of the reattachment process.

It is recalled that a number of modifications have been introduced to the baseline $\omega$ equation of Wilcox, particularly in relation to model constants, in an effort to achieve greater consistency between the two equations and to reduce the inappropriate tendency of the $\omega$ based model to return excessive recirculation. As is evident, the modified version continues to display this trend, although it must be born in mind that the model used here resolves anisotropy and introduces a substantially greater sensitivity to curvature, via the strain invariants in Eq. (6), relative to the linear model. Were Wilcox's w-equation to be used here, the recirculation length would be grossly excessive. Hence, the present $\omega$-equation has gone some way towards rectifying the aberrant behavior noted above. The frequent observation that Wilcox's LRN $\omega$-equation (Wilcox (1994)) performs quite differently to the earlier highReynolds-number form (Wilcox (1988)) in separated flow, suggests a high sensitivity to the nature of the LRN-model functions in the w-equation, and this may well be one source for the delayed reattachment observed here.

Figures 8 - 10 provide, for Models 1, 2 and 3, respectively, distributions of streamwise velocity, Reynolds-stresses and stress invariants at three streamwise positions, $x / H=2,6$ and 8. The first position is roughly in the middle of the recirculation zone, $x / H=6$ is a 
short distance behind the reattachment point, while $x / H=8$ cuts across the windward side of the hill where the flow is subjected to strong acceleration. These three locations may be claimed to represent regions in which three distinctly different types of flow conditions are encountered in the present configuration.

The velocity-profile plots in Figs. 8 - 10 show all three models to give a reasonable representation of the mean flow, with Model 3 providing somewhat better accord with the LES solution. All three models return a slightly insufficient rate of recovery after reattachment. While this might be expected in the case of Model 3, because of the delayed reattachment it predicts, this weakness is accentuated by Models 1 and 2, which give premature reattachment. Hence, Model 3 may be claimed to provide the best representation, despite the slightly delayed reattachment.

All three models also give similar shear-stress variations. At $x / H=2$, the predicted maximum shear stress is consistently too low. This would be expected to go hand-in-hand with an excessive shear strain. However, this link ignores the influence of the pressure gradient in the momentum balance, and it is not possible to uniquely relate the two. What is observed is that Model 3 gives the lowest level of shear stress and the highest level of shear strain. While the details of the mechanism by which the shear stress is generated are in principle, immaterial to the effect of the stress on the mean-flow field, Reynolds-averaged models are unable to account for the dynamic effects of the coherent motion and will therefore give an erroneous result in the shear layer. In particular, the predicted level is likely to be lower than that returned by the LES. The fact that the strain in the shear layer and the reattachment point are nevertheless broadly correct must, as noted already, be a consequence of compensating adjustments to the present field. As is seen from plots (f), the predicted shear stress at $x / H=8$ is much higher than the LES level. This is probably due to the significant contribution of shear-stress transport to the stress balance, which none of the present algebraic models are able to represent. Consistently, they suggest that the predicted turbulence energy is too low, again because of transport. In addition, the state of anisotropy prevailing at this location is rather exceptional, with the spanwise stress returned by the LES being extremely high, implying a mechanism which is not accounted for by the models, even not in a qualitative sense.

As regards the normal stresses at $x / H=2$ and 6 , all three models predict reasonable distributions and a broadly correct separation among the stresses, except for the region close 
to the wall. This emerges more clearly from plots (g) and (h), which show distributions of the anisotropy components. Near the lower wall, there are material differences between Model 2 and the other two models. It is recalled that this model does not include the wallorientation term in Eq. (15) and was not able to return the correct wall-limiting behavior $b_{22}=-1 / 3$ and $A=0$. This defect is also evident in Fig. $9(\mathrm{j}),(\mathrm{k})$. A clear reflection of the above differences in model characteristics is the different behavior of the $I I / I I I$-loci shown in plots $(\mathrm{m})$ and $(\mathrm{n})$. The LES results suggest that the turbulent state at $x / H=2$ and 6 , in the region $y / H<1$, is characterized by that encountered in homogeneous axisymmetric contraction, represented by the lower-left edge of the realizability triangle. This behavior is not reproduced by Model 2, but is returned - at least qualitatively correctly - by both Models 1 and 3. Since the non-dimensional shear parameters $S^{* *}$ and $\Omega^{* *}$ are considerably weaker in the separated region than in simple wall shear, the distributions of the invariants returned by Model 2 show unrealistic features, resulting in poor prediction of the near-wall anisotropy. In particular, the wall-normal turbulence is significantly too high.

\subsection{Plane impinging jet}

Results for this third test case are presented in Figs. 11 - 14. First, Fig. 11 provides a velocity-vector plot predicted by Model 1, alongside a comparison of streamwise velocity profiles at $y / D=4,6$ and 8 , all three sections being in the free-jet region. Differences among the predicted profiles are insignificant in comparison to those between predictions and simulation. All models overestimate the centerline velocity and underestimate the jet spreading rate, indicating defects in the formulation of $C_{B}$ (Eq. (10)) and/or the turbulent diffusion terms in turbulence-transport equations for free shear flows.

Figures 12 - 14 present, in parallel to the practice adopted in the previous case, profiles of velocity, Reynolds stresses, anisotropy components and anisotropy invariants at three positions: $x / D=0.04,1$ and 4 , where $x$ is the distance from the stagnation point along the lower wall. Each of the three figures relates to one of the models investigated. The three selected positions are, again, representative of distinct flow types; the first being in the impingement-dominated zone, the second in the highly curved portion in which the wall jet begins to form and the third being in the well-established wall-jet region.

All three models give rise to very similar velocity and shear-stress profiles. The predicted wall jet is narrower than that simulated, which is consistent with the lower magnitude of the 
shear stress returned by the models in the outer part of the free shear layer $y / D=1-1.5$. Closer to the wall, at $y / D=0.3-0.7$, the predicted shear stress at $x / D=1$ is seen to be much larger than the simulated level. In this region of intense curvature and high inertial effects, stress-transport is likely to be high, and errors in the representation of turbulence are most likely to be caused by the models. The fact that the shear strain at that position is also excessive, is consistent with the link between shear strain and turbulence generation, but also suggests that the misrepresentation of the mean flow at that position is a consequence of the upstream structure of the jet, coupled with the high convective transport associated with the impingement process. At $x / D=4$, the wall jet is fairly well established, convective transport is weak, and the correspondence between shear stress and shear strain is much closer, with all three models returning fairly good results.

The normal stresses, plots (d), (e) and (f) in Figs. 12 - 14, and hence the anisotropy components, plots $(\mathrm{g}),(\mathrm{h})$ and (i), are not very well resolved by any of the models, but there are clear differences in model performance and also some distinctly positive predictive aspects that are worth being highlighted. It is pointed out first that the transverse normal stress is extremely high in the impingement region, where the flow is subjected to high curvature and acceleration. This feature parallels closely that observed in the previous hill-flow case, at $x / H=8$, where the flow accelerates strongly along the windward side of the hill. None of the present models (nor any other RANS model the authors are aware of) is capable of capturing this feature.

The second point to highlight is that Models 1 and 3 reproduce correctly, as they do in earlier cases, the near-wall variation of the wall-normal anisotropy $b_{22}$ and its limiting wall value $-1 / 3$. In contrast, Model 2 fails to do so. The differences in model performance are especially striking at $x / D=0.04$. As seen in Fig. $13(\mathrm{~g})$ and $(\mathrm{j})$, the normal stresses predicted by Model 2 return to isotropic turbulence in the stagnation region near the wall, resulting in an excessive level of $\overline{v v}$, compared to that provided by Models 1 and 3. Although the separation among the anisotropy components is not satisfactory, it improves with distance away from the immediate impingement region, especially that returned by Models 1 and 3 . This is well brought out by the profiles of the stress invariants, plots (l). While the nearwall anisotropy is too high, as is reflected by the excessive values of $I I$ and $I I I$, the flatness parameter is reproduced well, and it correctly approaches zero at the wall itself. This is also the case at the impingement location, $x / H=0.04$, where Model 2 produces a serious 
misrepresentation of the stress field, giving a state of isotropy $(A=1)$ at the wall, as a natural consequence of the absence of the wall-related fragments in Eq. (15).

The realizability maps, plots $(\mathrm{m}),(\mathrm{n})$ and $(\mathrm{o})$, demonstrate that all three models conform to realizability constraints, even in the most taxing strain field associated with the impingement process. The simulation suggests that the outer part of the shear layer conforms broadly to the process of axisymmetric expansion, followed by a thin region complying with axisymmetric contraction and ending with an asymptotic decay towards two-component turbulence. Model 2 entirely fails to return these trends. While Models 1 and 3 do not give a quantitatively correct representation of the $I I / I I I$-loci either, they do - at least for $x / H=4$ - reproduce the steep rise of the magnitude of $I I$ and $I I I$ and the trend towards the two-component state at the lower wall.

The differences among the models in respect of the near-wall state they predict are especially important for heat-transfer computations. Within the framework of algebraic stress/strain and flux/temperature-gradient formulations, the heat-flux vector can be approximated, most generally, with Daly and Harlow's (1970) Generalized Gradient Diffusion Hypothesis. The performance of this approximation depends crucially on the stress field, however. Near the wall, $b_{22}$ (i.e., $\overline{v v}$ ) plays an especially important role. A misrepresentation of this component at impingement and reattachment points can give rise to errors in the wall heat-transfer coefficient in excess of $100 \%$. Therein lies the importance of the distinction between Model 2 and Models 1 and 3. The differences observed here reinforce the conclusion that a model relying entirely on strain and vorticity invariants for the representation of anisotropy, as most NLEVMs do, cannot return the correct near-wall behavior.

\section{Concluding Remarks}

New modeling elements have been proposed as additional fragments to non-linear strain- and vorticity-dependent constitutive equations for the Reynolds stresses, with the objective of improving the representation of stress anisotropy in wall-bounded flows. These have been combined with two alternative length-scale-governing equations for dissipation and specific dissipation, respectively. Computational results were presented for three test cases involving, inter alia, wall-shear, separation and strong normal straining. The main conclusions derived from the study are as follows: 
- A constitutive stress-governing algebraic equation containing only velocity gradients, as a combination of strain and vorticity terms, is insufficient to achieve a credible representation of near-wall anisotropy in the presence of weak shear and strong normal straining associated with impingement and reattachment.

- In order to secure a realistic representation of near-wall anisotropy, the principal predictive requirement is for the model to reproduce the damping effect of the wall on the turbulent stresses, regardless of the strength of the shear rate. A correct representation of the wall-normal intensity is a pre-requisite for a correct asymptotic behavior of turbulence towards a state of two-component turbulence at the wall.

- The use of the specific dissipation $\omega$ can offer significant benefits in separated flow, but the related equation must be carefully formulated and calibrated, with close reference to the dissipation-rate equation, so as to avoid an excessive sensitivity to adverse pressure gradient, anomalous delay in reattachment and aberrant behavior at the edge of the shear layers bordering a free stream.

- While the present model proposals give improved predictions for near-wall turbulence, they do not alleviate problems arising from the influence of stress transport in strongly accelerating and decelerating flows. This requires the use of second-moment closure or at least the introduction of approximations of stress-anisotropy transport.

\section{References}

Abe, K., Kondoh, T. and Nagano, Y. 1997. On Reynolds stress expressions and near-wall scaling parameters for predicting wall and homogeneous turbulent shear flows. Int. J. Heat Fluid Flow, 18, 266-282.

Abe, K. and Suga, K. 2001a. Toward the development of a Reynolds-averaged algebraic turbulent scalar-flux model. Int. J. Heat Fluid Flow, 22, 19-29.

Abe, K. and Suga, K. 2001b. Large eddy simulation of passive scalar in complex turbulence with flow impingement and flow separation. Heat Transfer Asian Research, 30, 402-418.

Apsley, D.D. and Leschziner, M.A. 1998. A new low-Reynolds-number nonlinear two-equation turbulence model for complex flows. Int. J. Heat and Fluid Flow, 19, 209-222. 
Apsley, D.D. and Leschziner, M.A. 2000. Advanced turbulence modelling of separated flow in a diffuser. Flow, Turbulence and Combustion, 63, 81-112.

Craft, T.J., Launder, B.E. and Suga, K. 1996. Development and application of a cubic eddyviscosity model of turbulence. Int. J. Heat and Fluid Flow, 17, 108-115.

Craft, T.J. and Launder, B.E. 1996. A Reynolds stress closure designed for complex geometries. Int. J. Heat and Fluid Flow, 17, 245-000.

Daly, B.J. and Harlow, F.H. 1970. Transport equations in turbulence. Phys. Fluids, 13, 2634-2649.

Durbin, P.A. 1993. Application of a near-wall turbulence model to boundary layers and heat transfer. Int. J. Heat and Fluid Flow, 14, 316-323.

Gatski, T.B. and Speziale, C.G. 1993. On explicit algebraic stress models for complex turbulent flows. J. Fluid Mech., 254, 59-78.

Iacovides, H. and Raisee, M. 1997. Computation of flow and heat transfer in 2D rib roughened passages. Proc. 2nd Int. Symp. On Turbulence, Heat and Mass Transfer, Hanjalic, K. and Peters, T.W.J. (Eds.), Delft University Press, 21-30.

Jakirlic, S. and Hanjalic, K. 1995. A second-moment closure for non-equilibrium and separating high- and low-Re-number flows. Proc. 10th Symposium on Turbulent Shear Flows, Pennsylvania State, August, 23.25.

Jakirlic, S. et al. 2001. 9th ERCOFTAC/IAHR/COST workshop on refined turbulence modeling, Darmstadt, Test case 9.2.

Kim, J. et al. 1990. The collaborative testing of turbulence models (Organized by P. Bradshaw et al.), Data disk No. 4.

Lien, F.S. and Leschziner, M.A. 1994a. A general non-orthogonal collocated finite volume algorithm for turbulent flow at all speeds incorporating second-moment turbulence-transport closure, Part 1: Computational implementation. Comput. Methods Appl. Mech. Engrg, 114, 123-148.

Lien, F.S. and Leschziner, M.A. 1994b. Upstream monotonic interpolation for scalar transport with application to complex turbulent flows. Int. J. Num. Meths. In Fluids, 19, $527-548$. 
Menter, F.R. 1994. Two-equation eddy-viscosity turbulence models for engineering applications. AIAA J., 32, 1598-1605.

Nagano, Y. and Shimada, M. 1995. Rigorous modeling of dissipation-rate equation using direct simulations, Int. J. Japan Soc. Mech. Engrs: Ser. II, 38, 51-59.

Suga, K. 1995. Development and application of a non-linear eddy viscosity model sensitized to stress and strain invariants. Ph.D Thesis, (UMIST).

Suga, K. and Abe, K. 2000. Nonlinear eddy viscosity modeling for turbulence and heat transfer near wall and shear free boundaries. Int. J. Heat Fluid Flow, 21, 37-48.

Temmerman, L. and Leschziner, M.A. 2001. Large eddy simulation of separated flow in a streamwise periodic channel construction. Proc. 2nd Symposium on Turbulence and Shear Flow Phenomena, Stockholm, June, 27-29.

Temmerman, L., Leschziner, M.A., Mellen, C.P. and Froehlich, J. 2002. Investigation of wallfunction approximations and subgrid-scale models in Large Eddy Simulation of separated flow in a channel with streamwise periodic constrictions. Int. J. Heat Fluid Flow, (in press).

Wallin, S. and Johansson A.V. 2000. An explicit algebraic Reynolds stress model for incompressible and compressible turbulent flows. J. Fluid Mech., 403, 89-132.

Wilcox, D.C. 1988. Reassessment of the scale-determining equation for advanced turbulence models. AIAA J., 26, 1299-1310.

Wilcox, D.C. 1994. Simulation of transition with a two-equation turbulence model. AIAA J., 32, 247-255. 


\section{Figure captions}

1 Model performance in homogeneous strain: (a) Pure shear strain; (b) Plane strain; (c) Axisymmetric contraction; (d) Axisymmetric expansion.

2 Hill-flow geometry.

3 Plane-impinging-jet geometry.

4 Predictions of Model 1 for plane channel flow: (a) Mean velocity; (b) Reynolds stresses; (c) Budget of turbulence energy; (d) Anisotropy profiles; (e) Invariants of anisotropy tensor; (f) Invariant map.

5 Predictions of Model 2 for plane channel flow: (a) Mean velocity; (b) Reynolds stresses; (c) Budget of turbulence energy; (d) Anisotropy profiles; (e) Invariants of anisotropy tensor; (f) Invariant map.

6 Predictions of Model 3 ( $k$ - $\omega$ version) for plane channel flow: (a) Mean velocity; (b) Reynolds stresses; (c) Budget of turbulence energy; (d) Anisotropy profiles;

(e) Invariants of anisotropy tensor; (f) Invariant map.

$7 \quad$ Streamfunction contours for hill flow: (a) LES data; (b) Model 1; (c) Model 2; (d) Model 3 ( $k-\omega$ version).

8 Predictions of Model 1 for hill flow: (a)-(c) Mean velocity; (d)-(f) Reynolds stresses; (g)-(i) Anisotropy profiles; (j)-(l) Invariants of anisotropy tensor; (m)(o) Invariant map in the lower-wall region.

9 Predictions of Model 2 for hill flow: (a)-(c) Mean velocity; (d)-(f) Reynolds stresses; (g)-(i) Anisotropy profiles; (j)-(l) Invariants of anisotropy tensor; (m)(o) Invariant map in the lower-wall region.

10 Predictions of Model 3 ( $k-\omega$ version) for hill flow: (a)-(c) Mean velocity; (d)(f) Reynolds stresses; (g)-(i) Anisotropy profiles; (j)-(l) Invariants of anisotropy tensor; (m)-(o) Invariant map in the lower-wall region.

11 Predictions in free-jet region of impinging jet: (a) Velocity vectors by Model 1; (b) Mean velocity at $y / D=8 ;$ (c) $y / D=6$; (d) $y / D=4$.

12 Predictions of Model 1 in near-wall region of impinging jet: (a)-(c) Mean velocity; (d)-(f) Reynolds stresses; (g)-(i) Anisotropy profiles; (j)-(l) Invariants of anisotropy tensor; (m)-(o) Invariant map in the near-wall region.

13 Predictions of Model 2 in near-wall region of impinging jet: (a)-(c) Mean velocity; (d)-(f) Reynolds stresses; (g)-(i) Anisotropy profiles; (j)-(l) Invariants of anisotropy tensor; (m)-(o) Invariant map in the near-wall region. 
14 Predictions of Model 3 ( $k$ - $\omega$ version) in near-wall region of impinging jet: (a)(c) Mean velocity; (d)-(f) Reynolds stresses; (g)-(i) Anisotropy profiles; (j)-(l) Invariants of anisotropy tensor; (m)-(o) Invariant map in the near-wall region. 


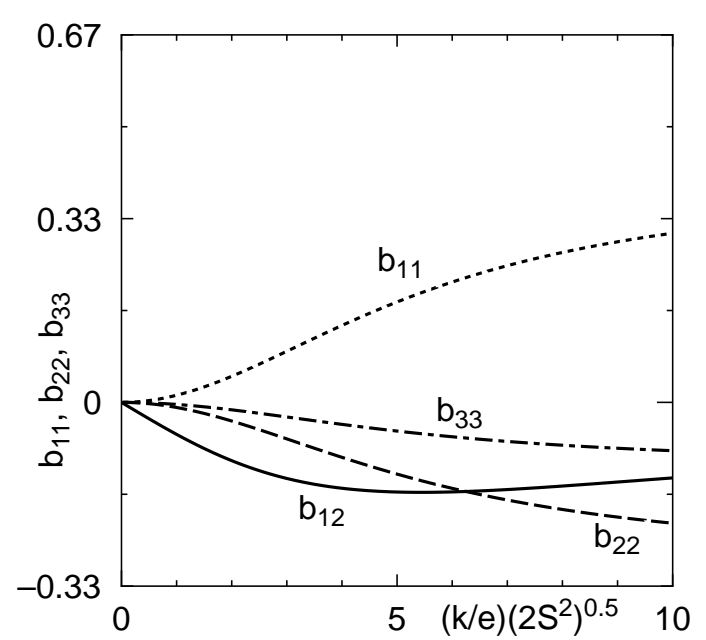

(a)

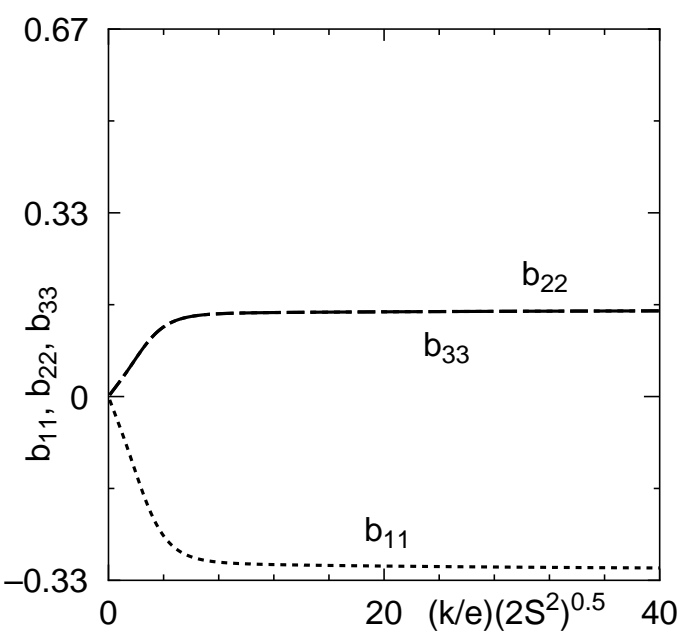

(c)

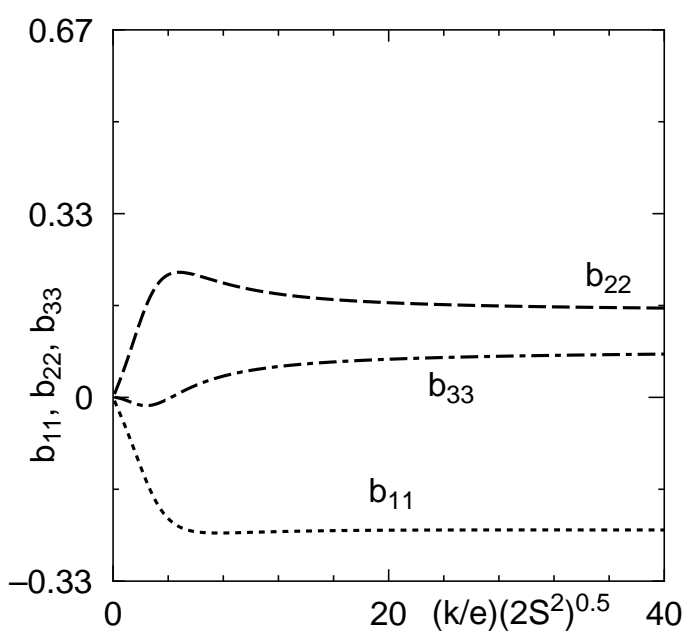

(b)

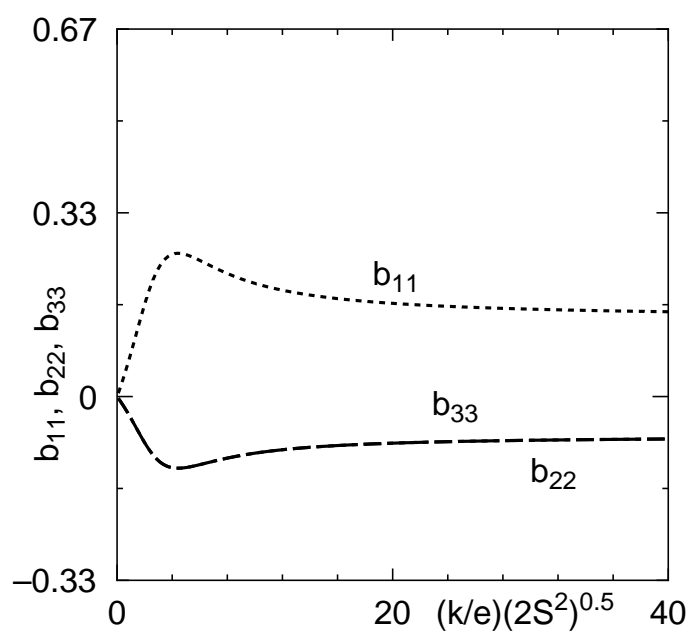

(d)

Figure 1: $\quad$ «Abe et al.» 
Wal I

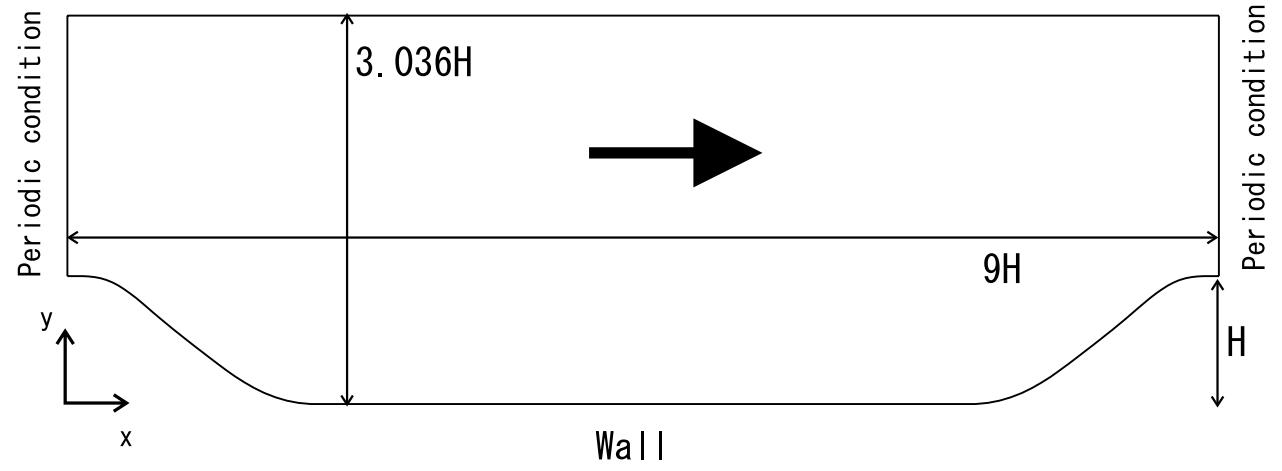

Figure 2: $\quad \ll$ Abe et al.» 


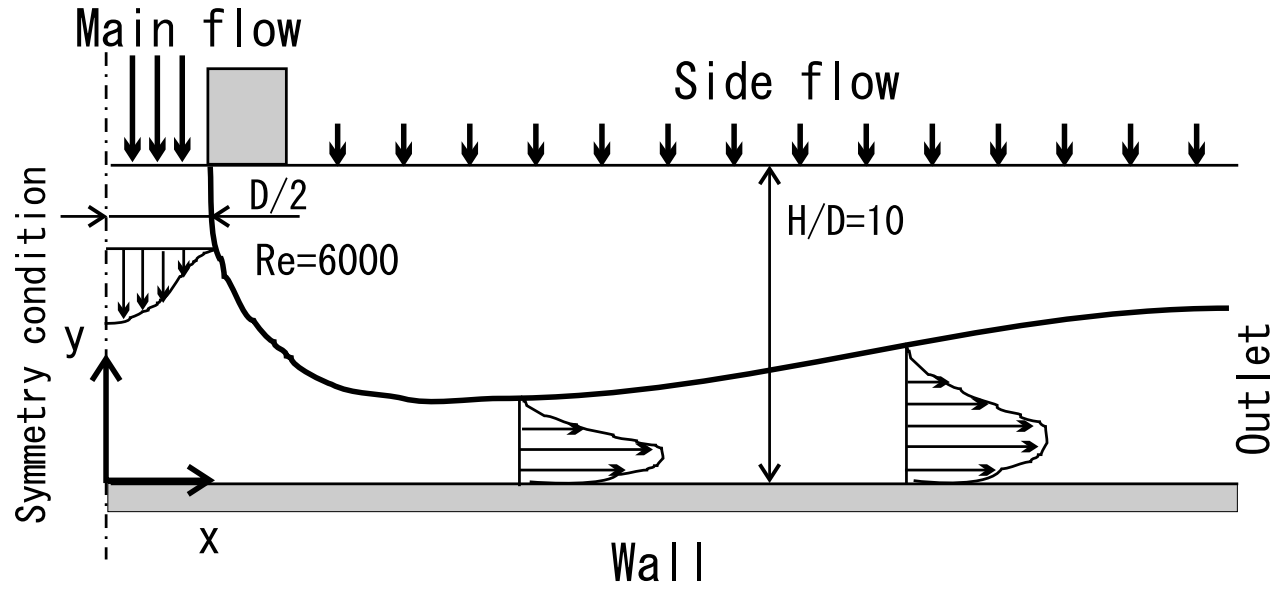

Figure 3: $\quad \ll$ Abe et al.» 


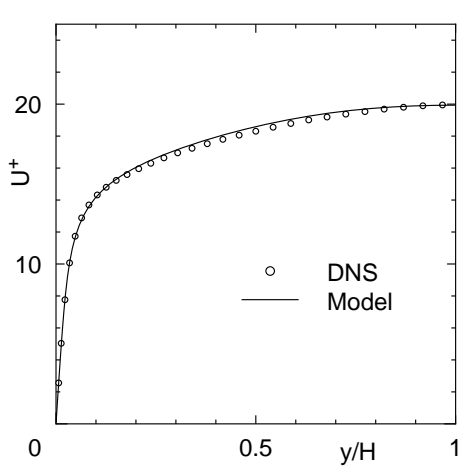

(a)

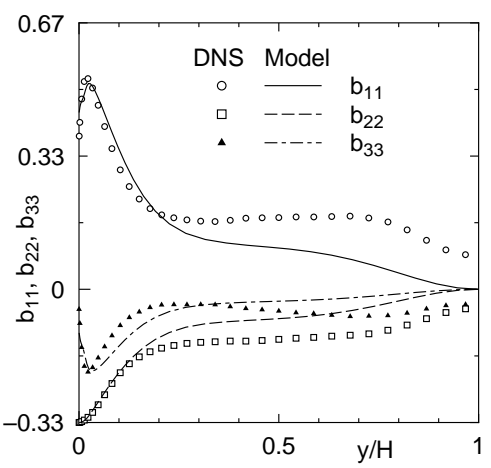

(d)

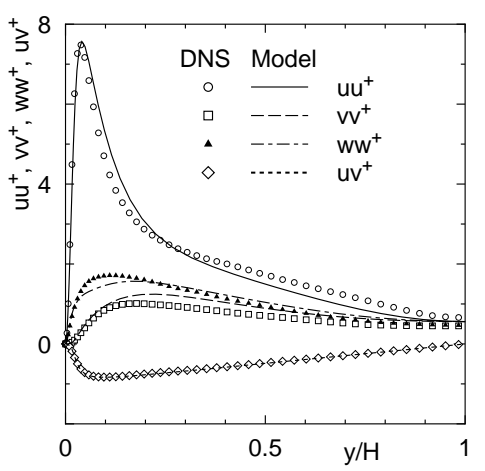

(b)

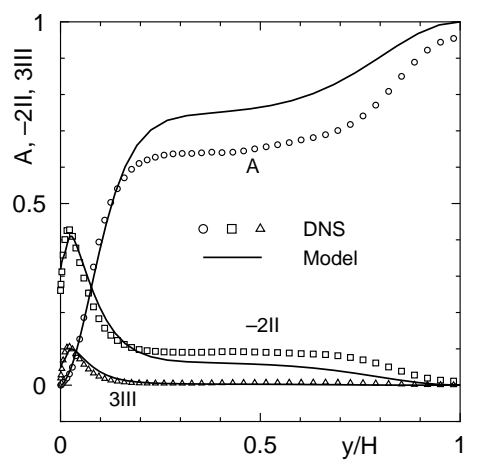

(e)

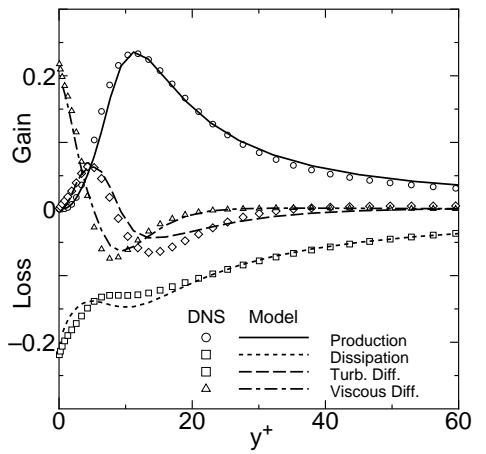

(c)

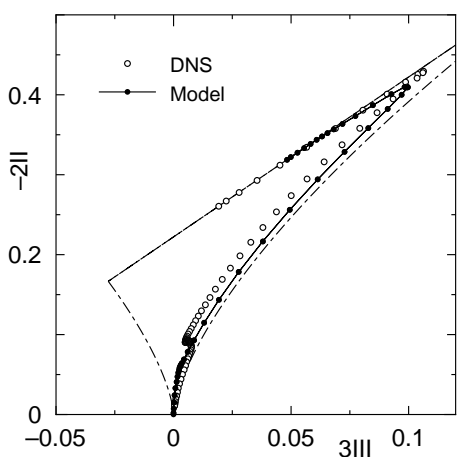

(f)

Figure 4: $\quad \ll$ Abe et al.» 


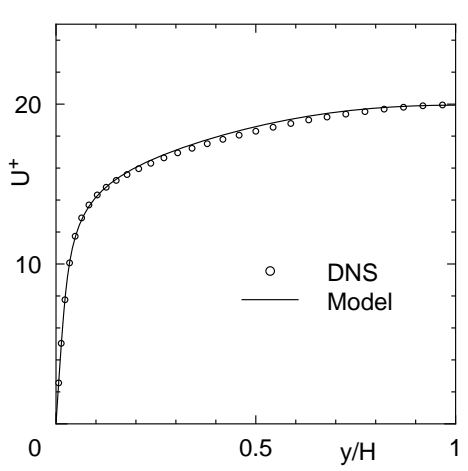

(a)

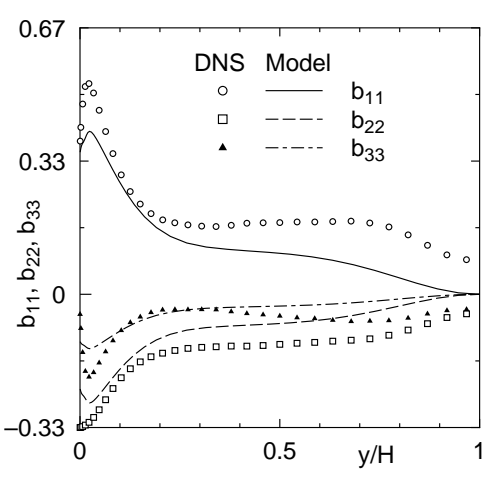

(d)

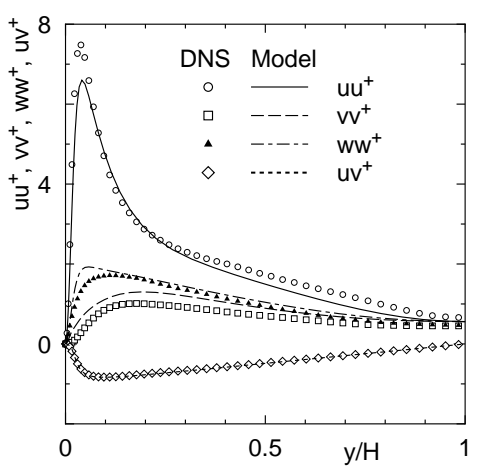

(b)

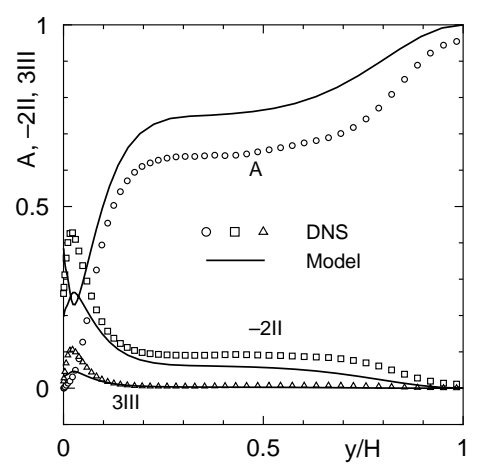

(e)

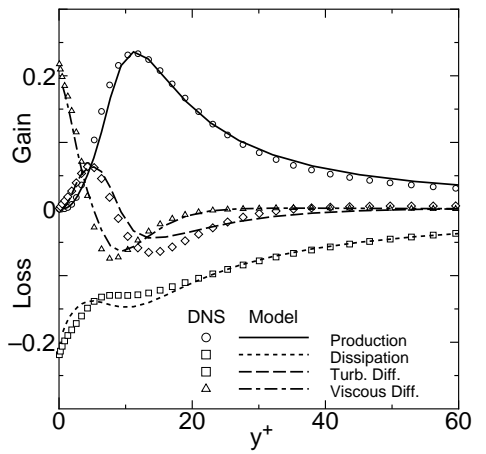

(c)

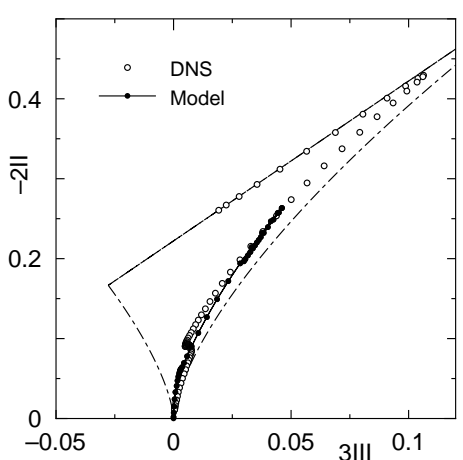

(f)

Figure 5: $\quad \ll$ Abe et al.» 


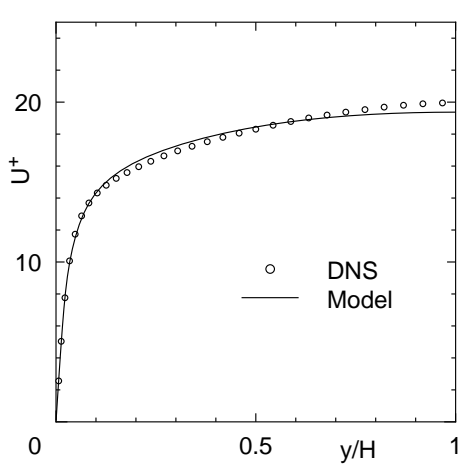

(a)

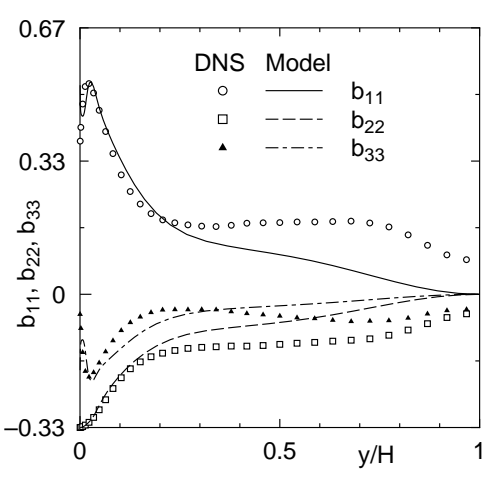

(d)

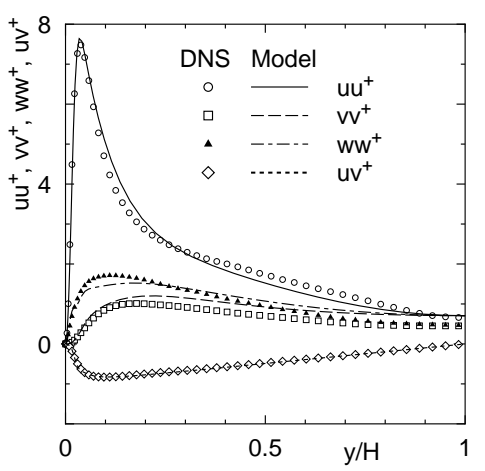

(b)

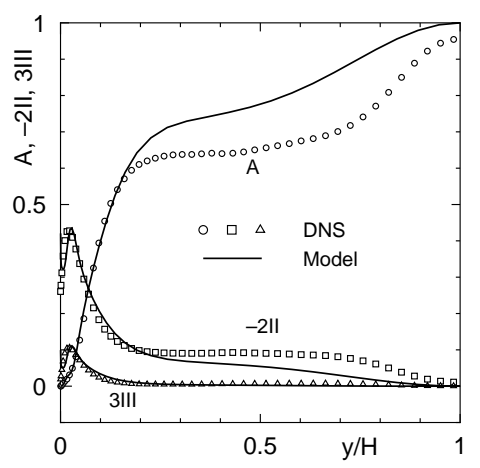

(e)

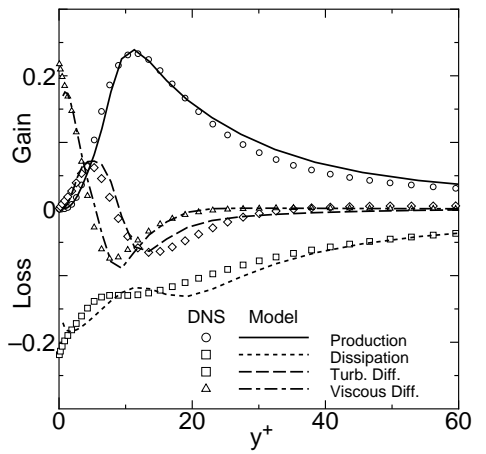

(c)

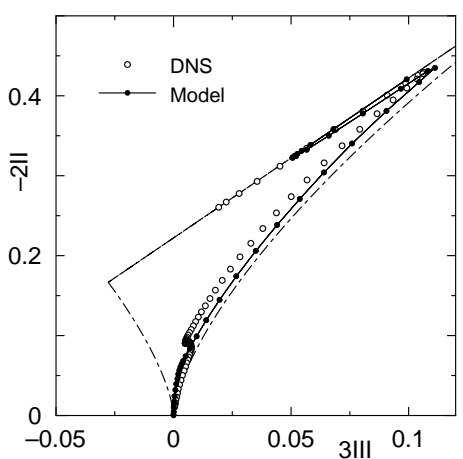

(f)

Figure 6: $\quad \ll$ Abe et al.» 


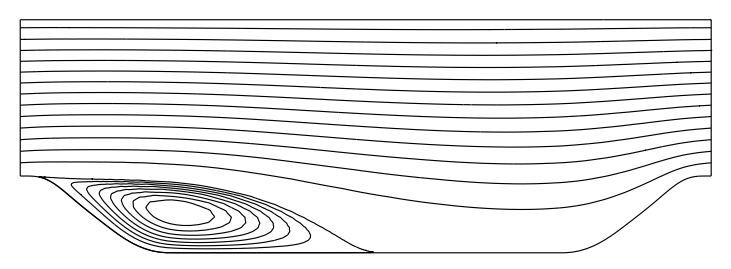

(a)

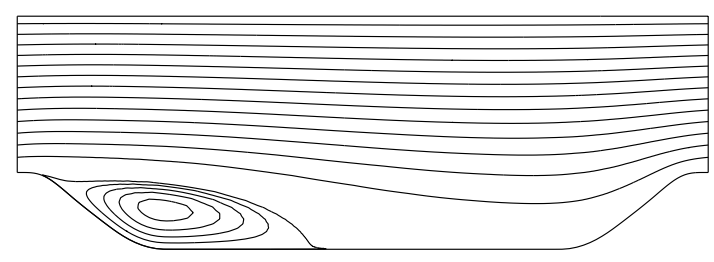

(c)

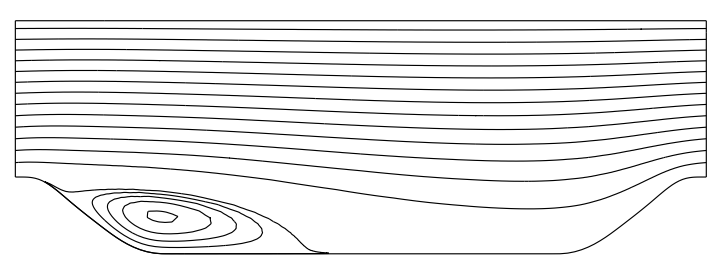

(b)

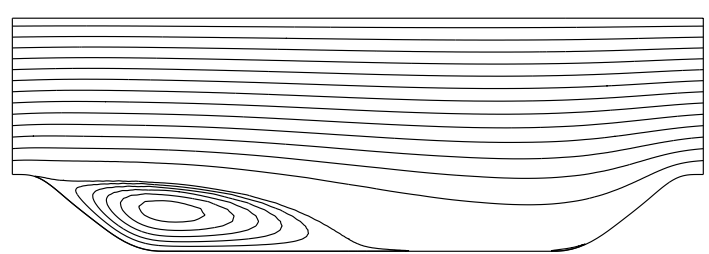

(d)

Figure 7:

«Abe et al.» 


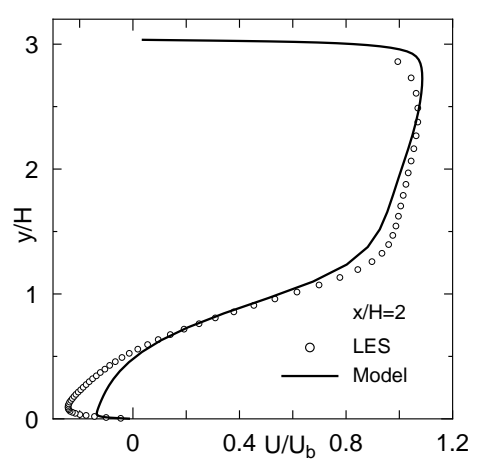

(a)

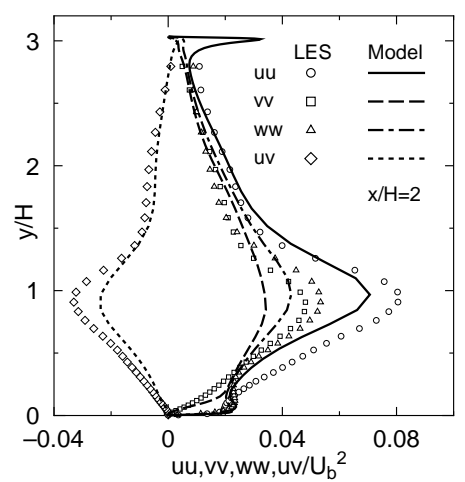

(d)

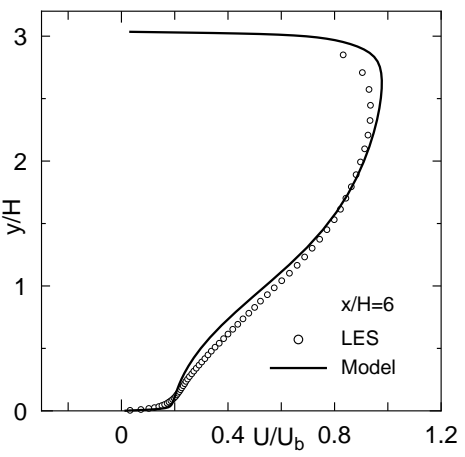

(b)

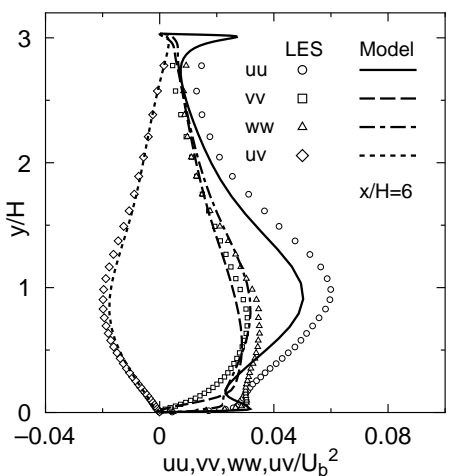

(e)

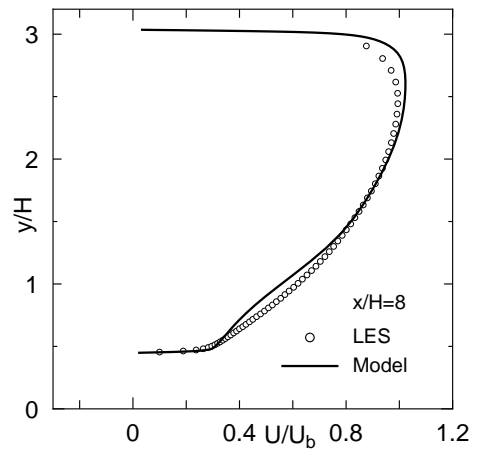

(c)

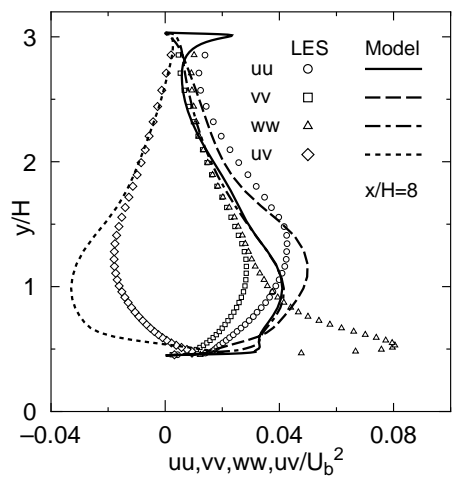

(f)

Figure 8:

«Abe et al.» 


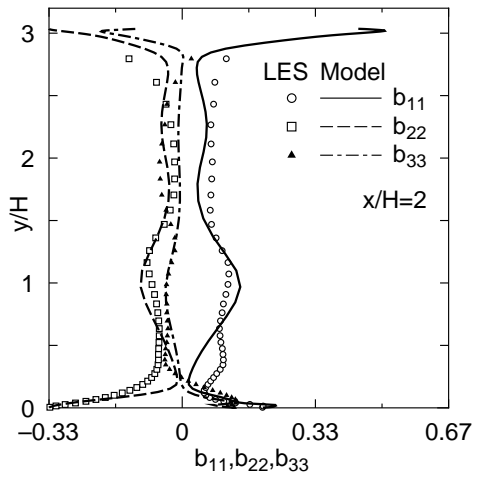

(g)

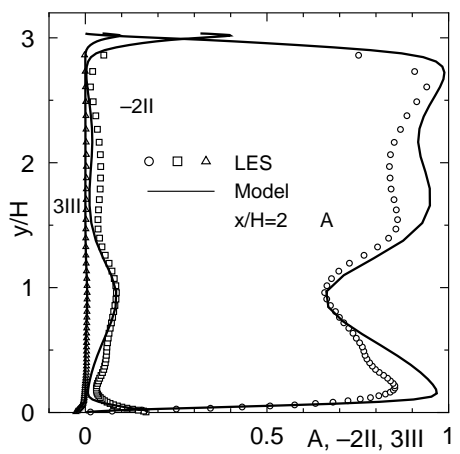

(j)

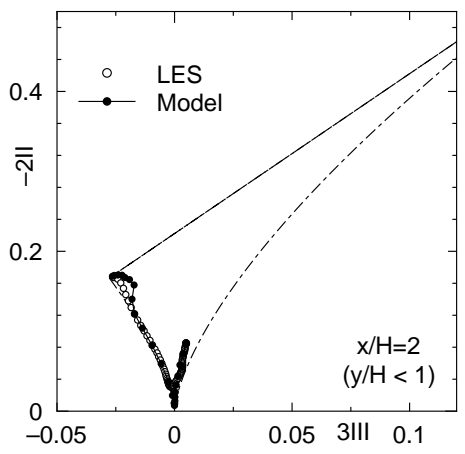

(m)

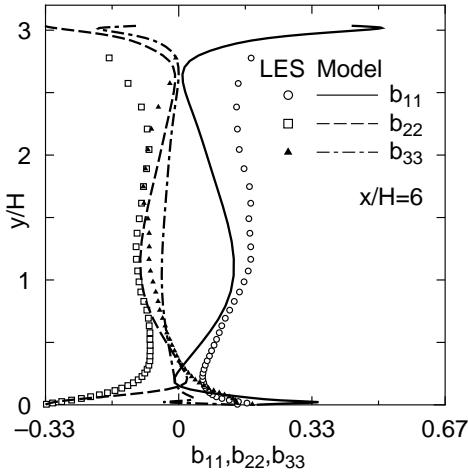

(h)

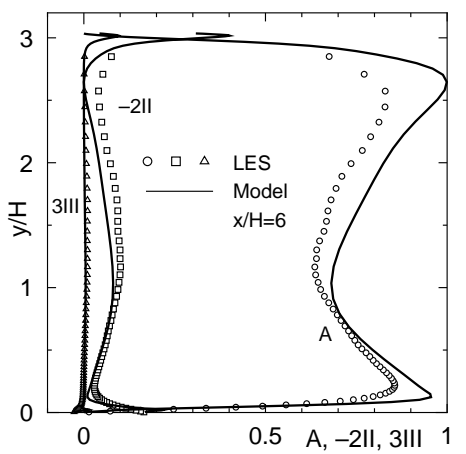

(k)

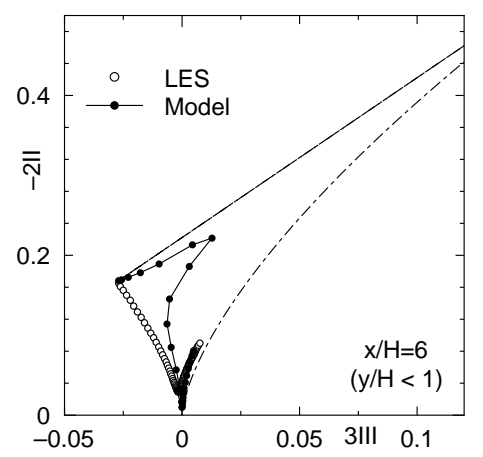

(n)

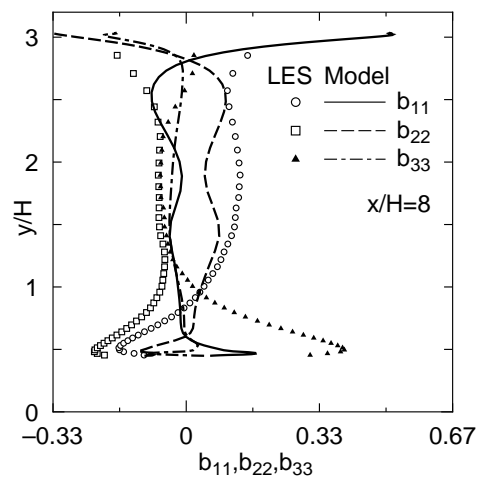

(i)

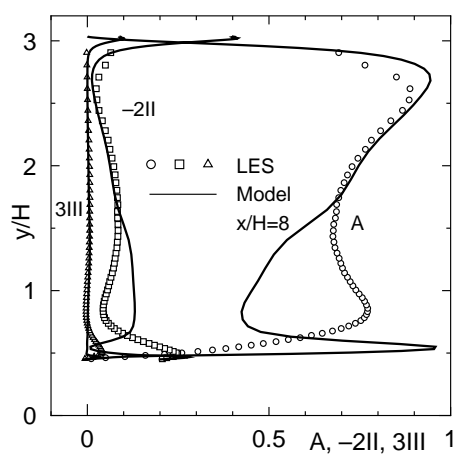

(1)

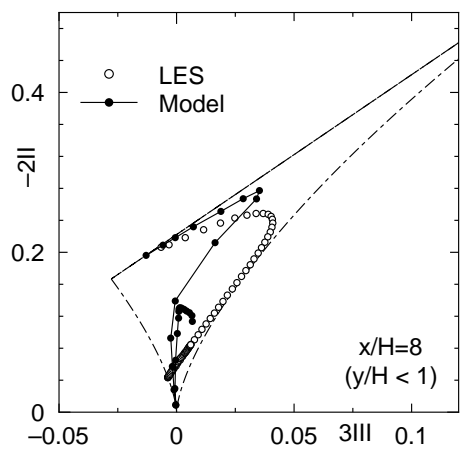

(o)

Figure 8: (continued)

«Abe et al.» 


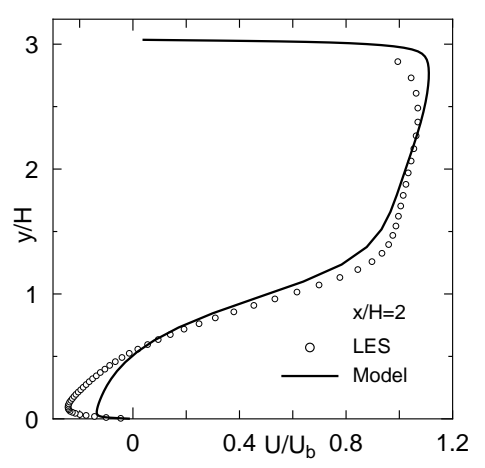

(a)

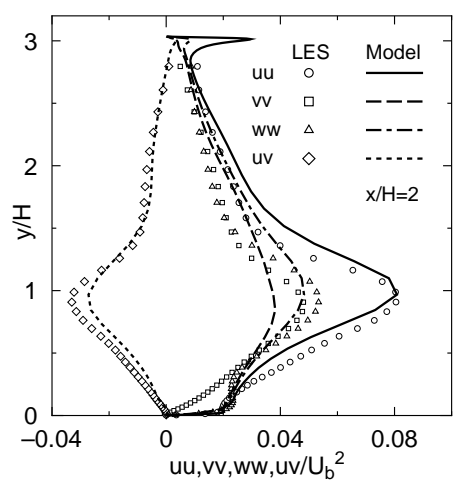

(d)

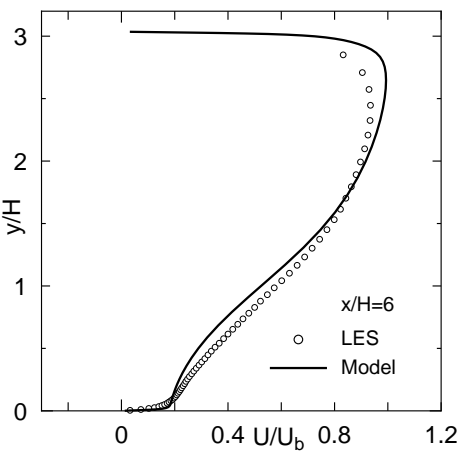

(b)

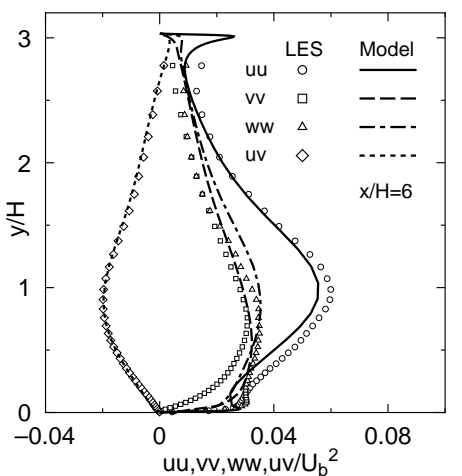

(e)

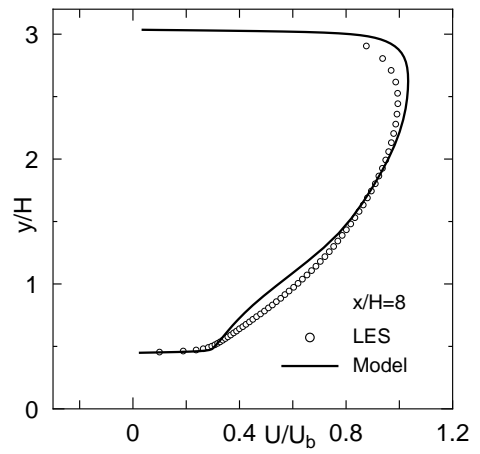

(c)

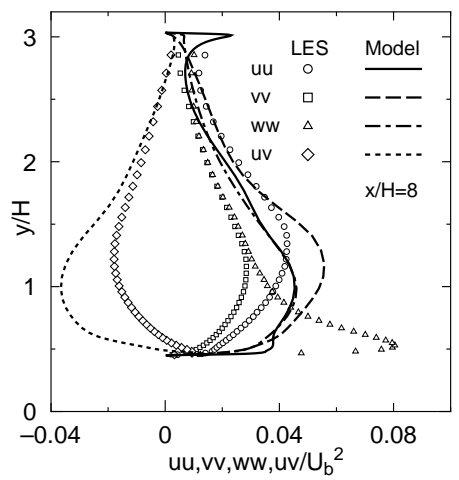

(f)

Figure 9:

«Abe et al.» 


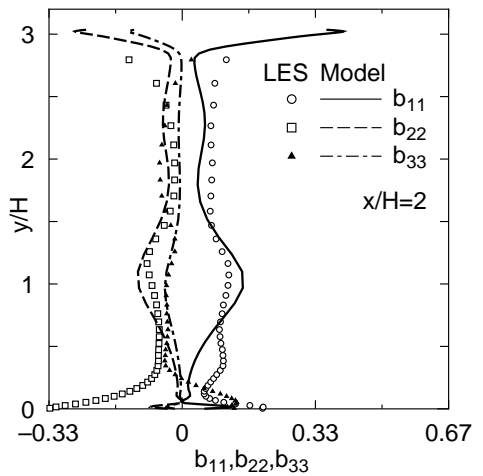

(g)

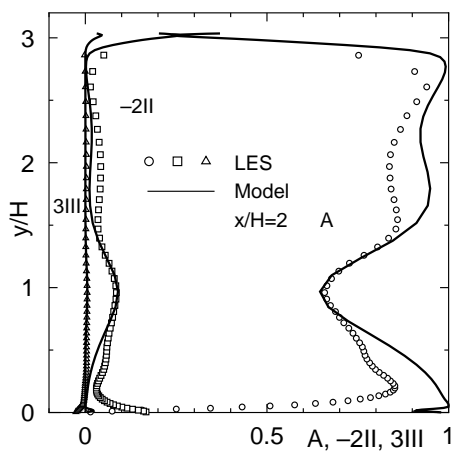

(j)

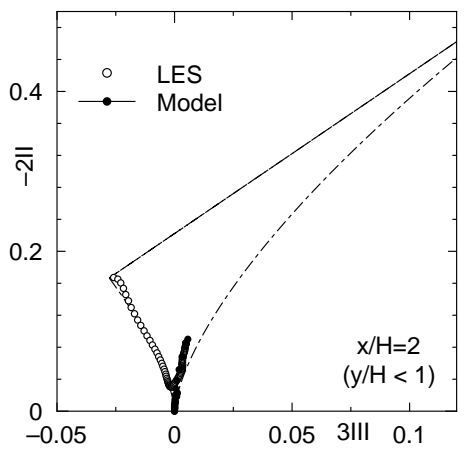

(m)

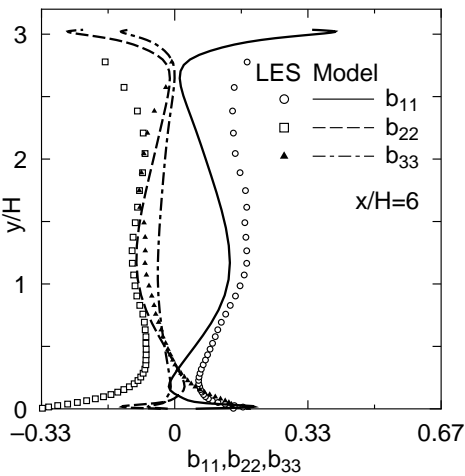

(h)

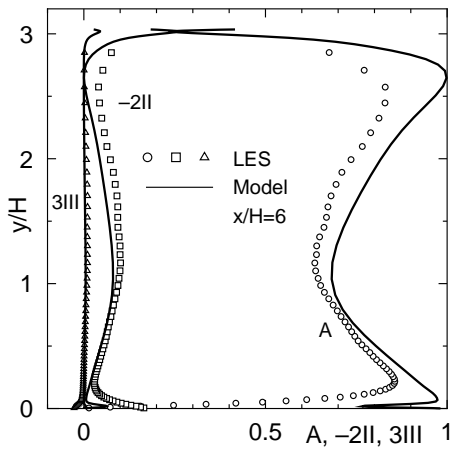

(k)

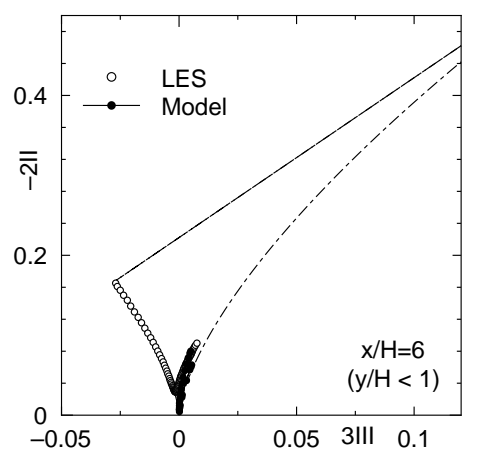

(n)

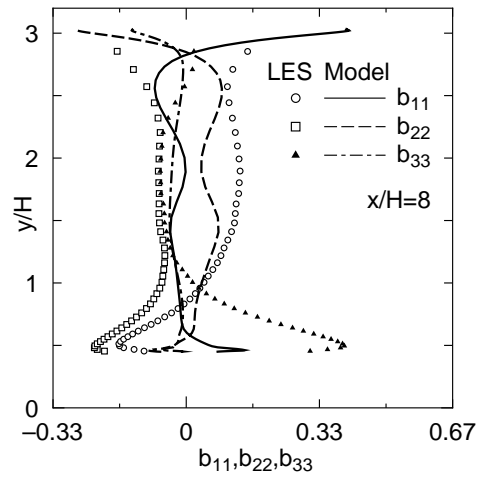

(i)

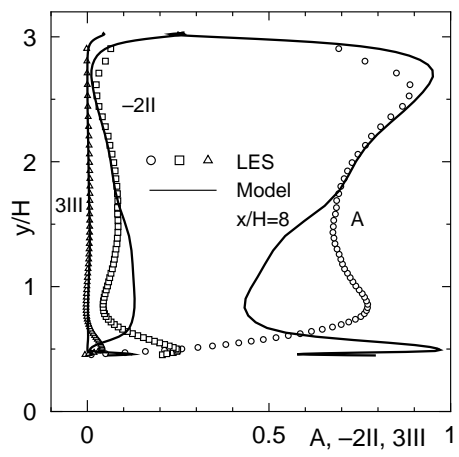

(1)

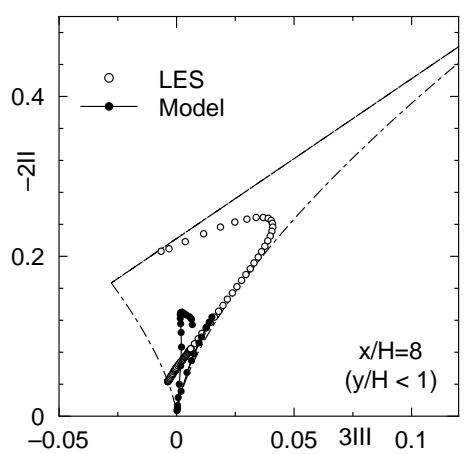

(o)

Figure 9: (continued)

«Abe et al.» 


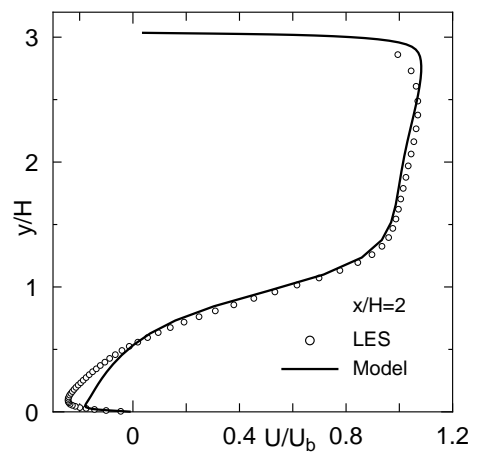

(a)

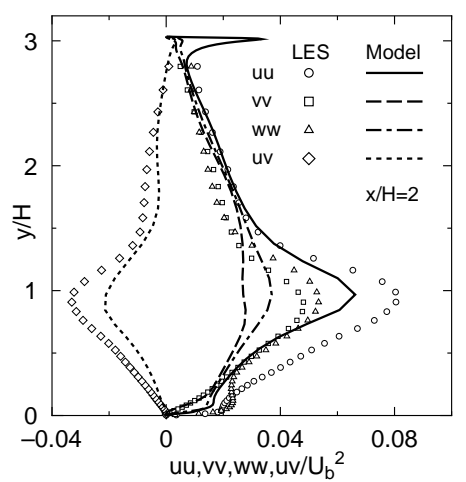

(d)

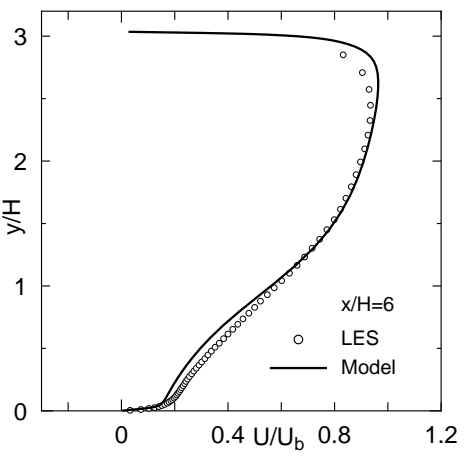

(b)

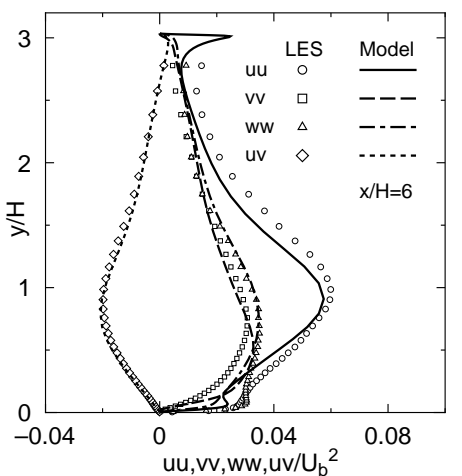

(e)

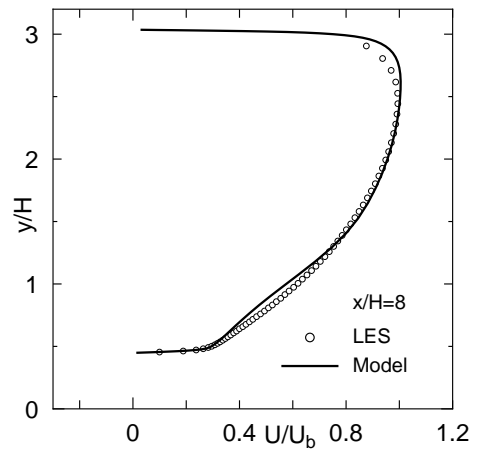

(c)

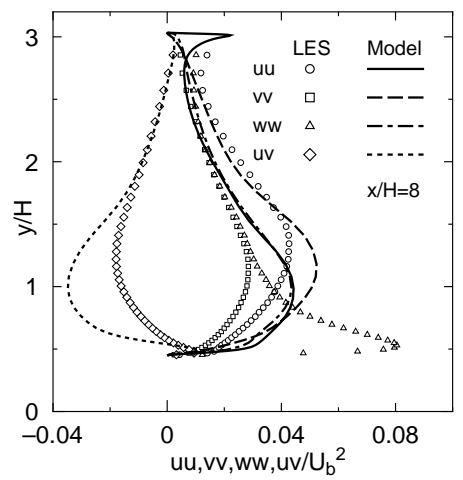

(f)

Figure 10:

«Abe et al.» 


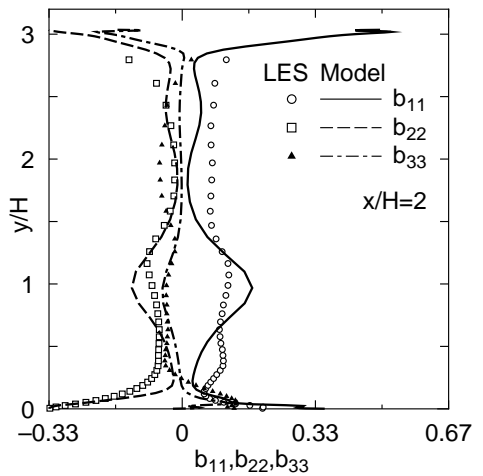

(g)

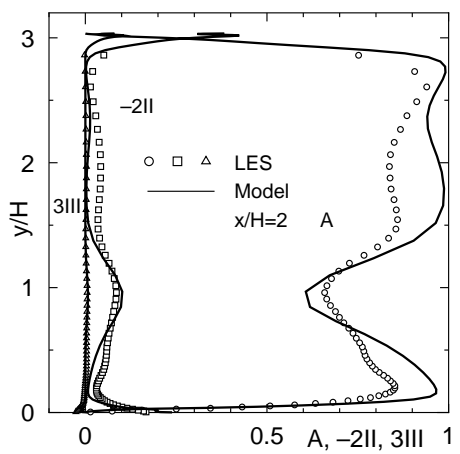

(j)

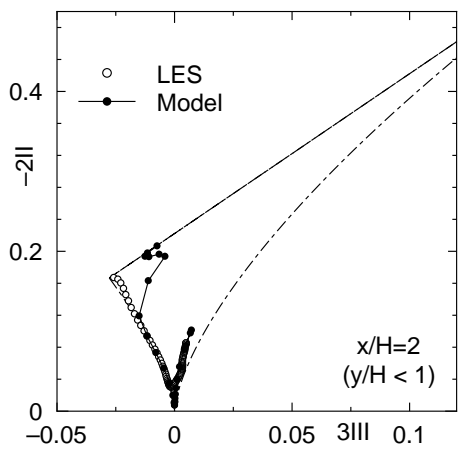

(m)

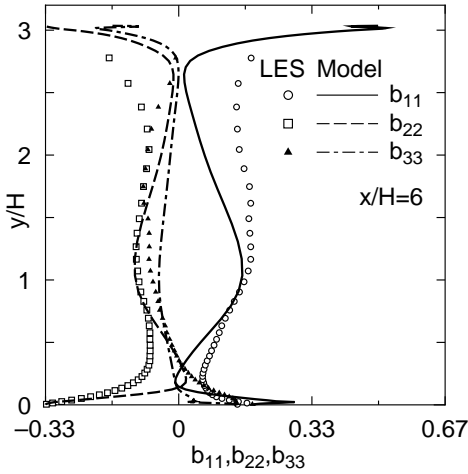

(h)

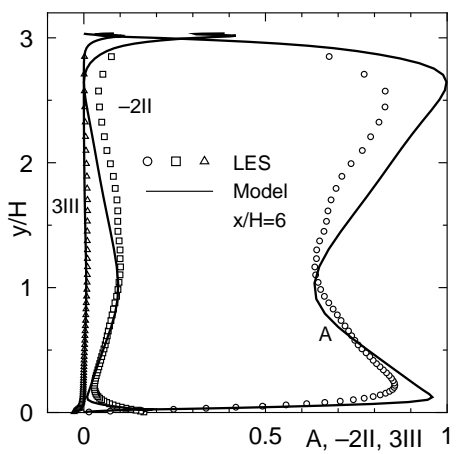

(k)

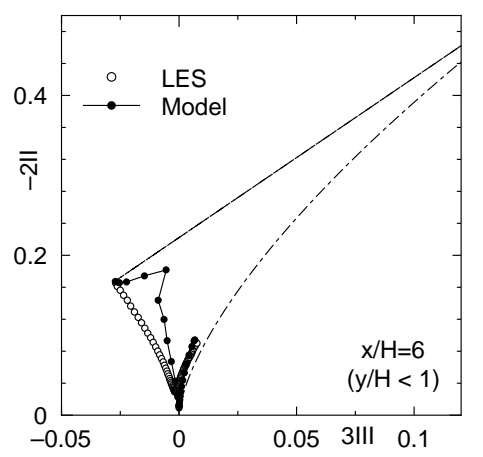

(n)

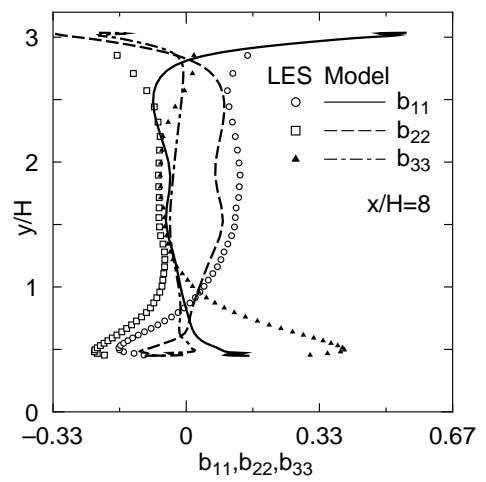

(i)

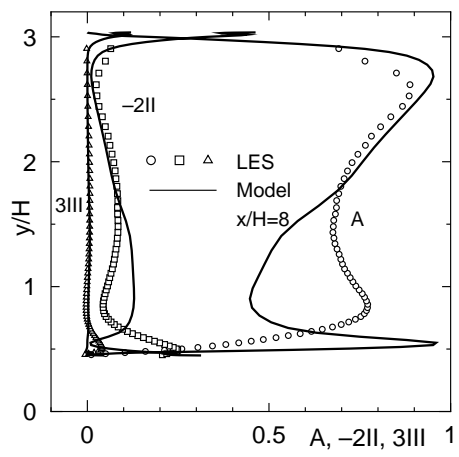

(1)

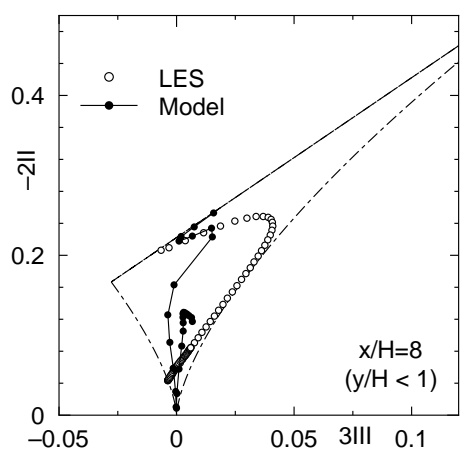

(o)

Figure 10: (continued)

«Abe et al.» 


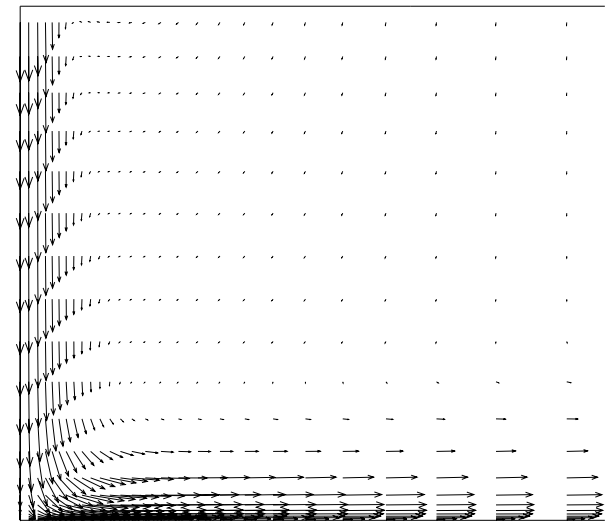

(a)

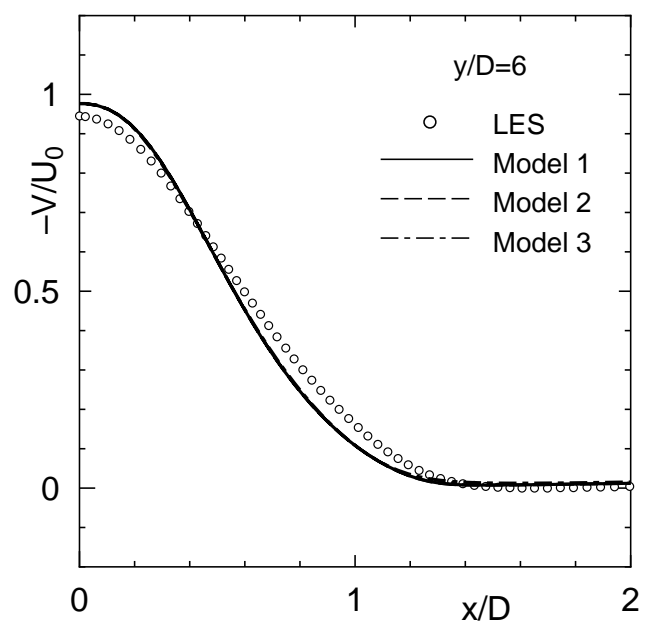

(c)

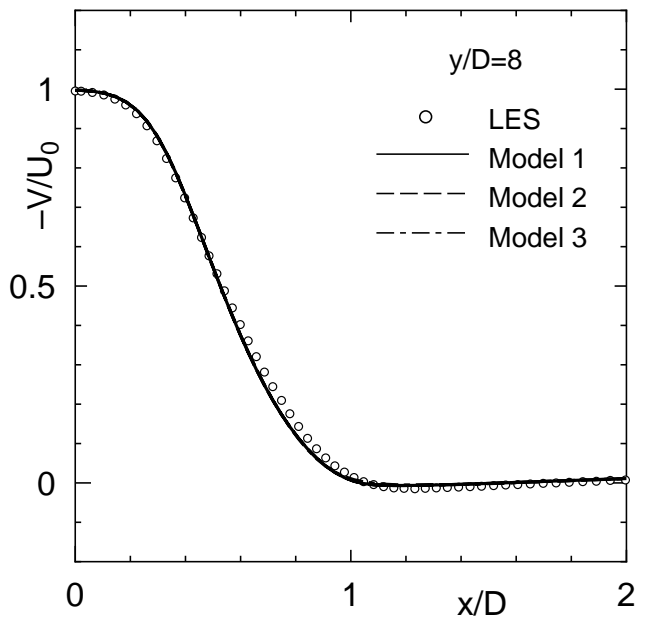

(b)

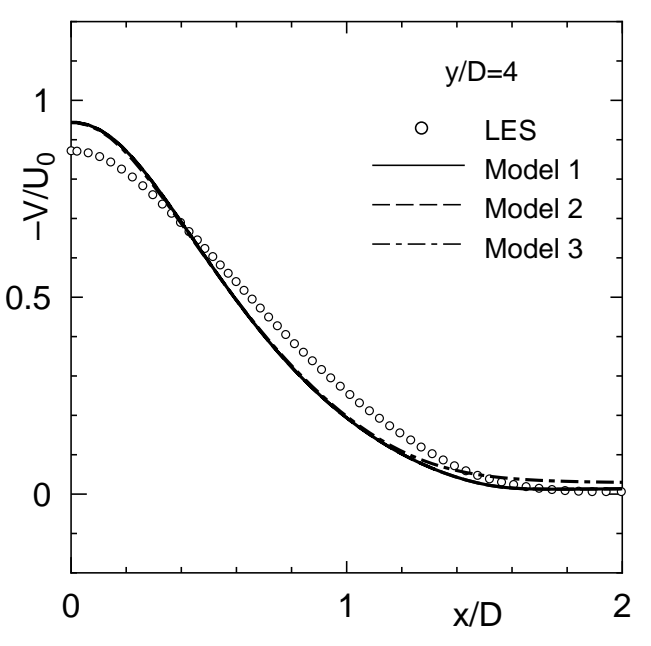

(d)

Figure 11: $\quad$ «Abe et al.» 


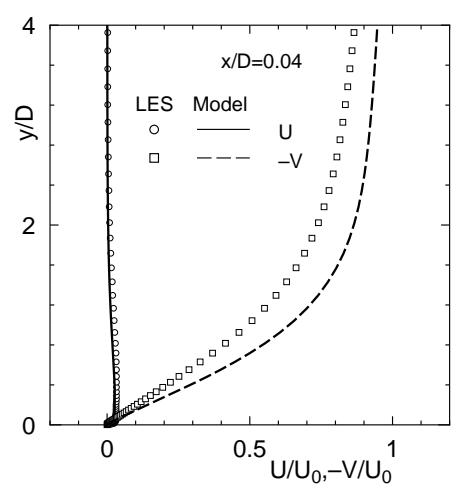

(a)

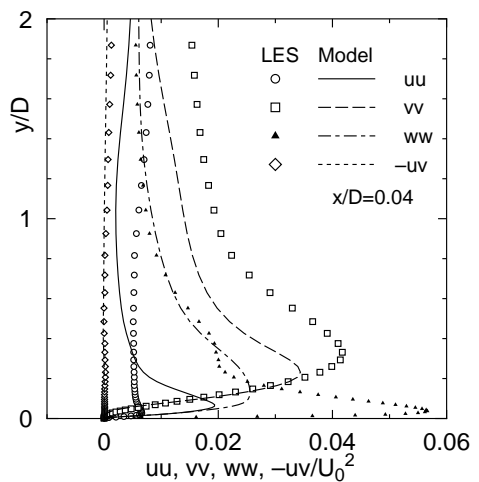

(d)

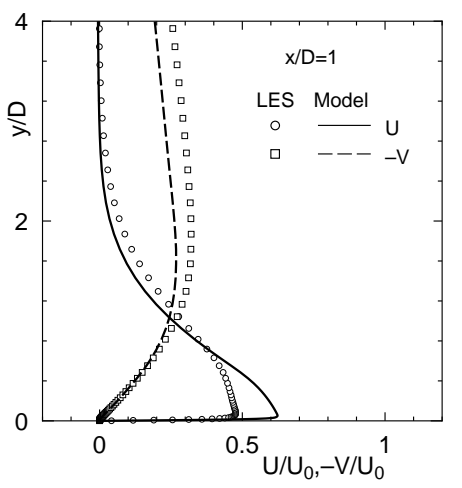

(b)

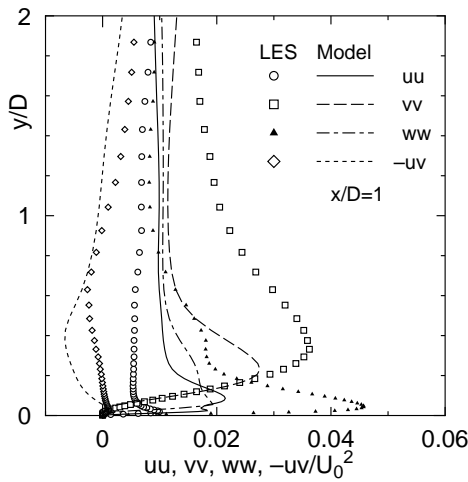

(e)

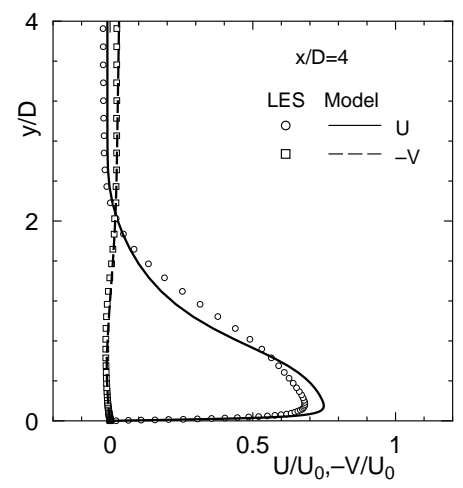

(c)

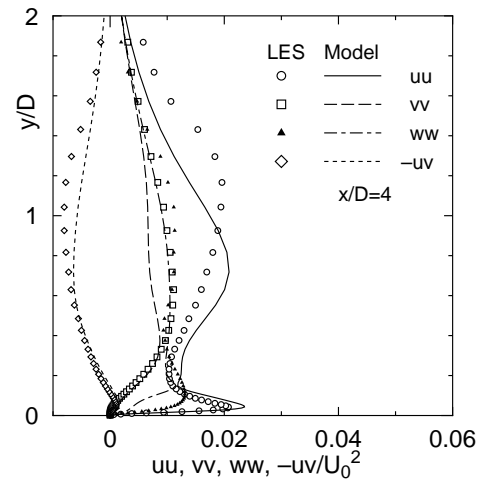

(f)

Figure 12:

$\ll$ Abe et al.» 


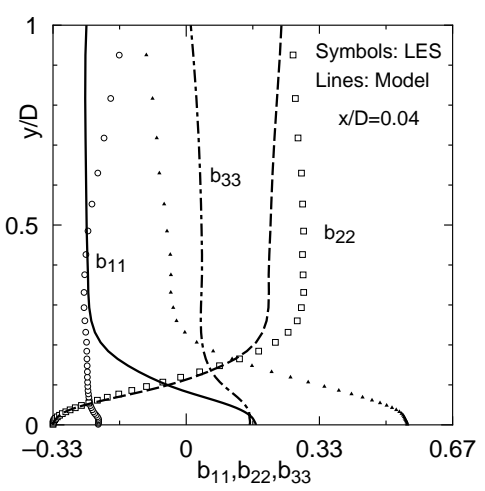

(g)

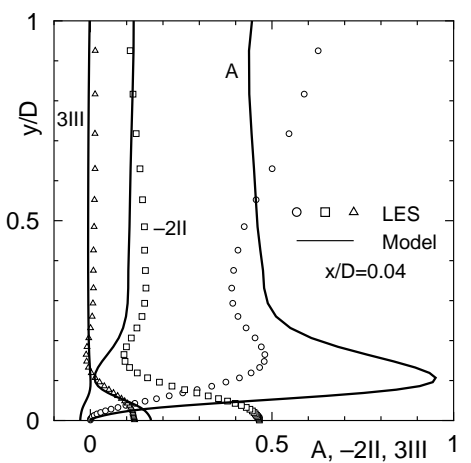

(j)

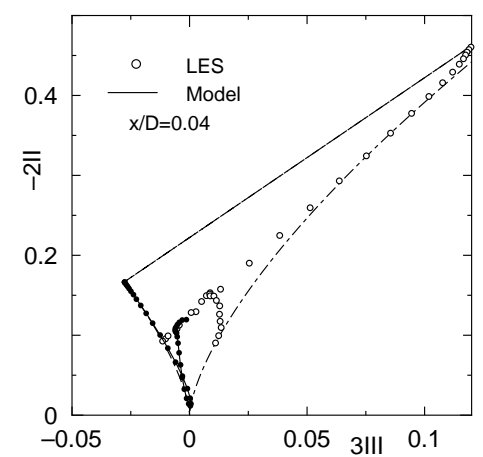

(m)

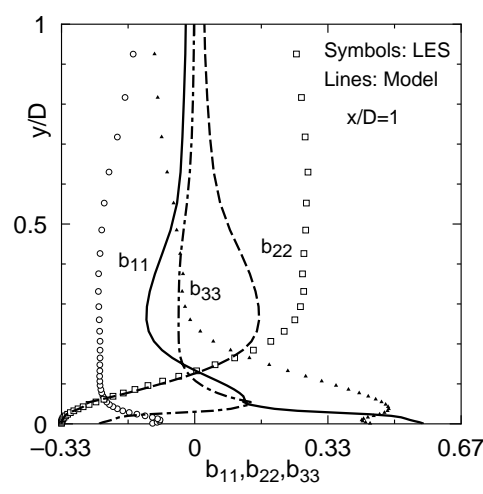

(h)

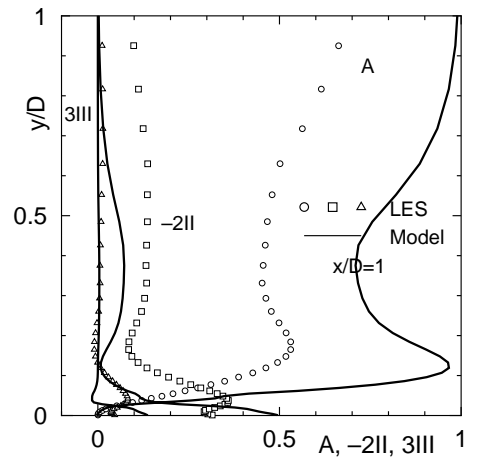

(k)

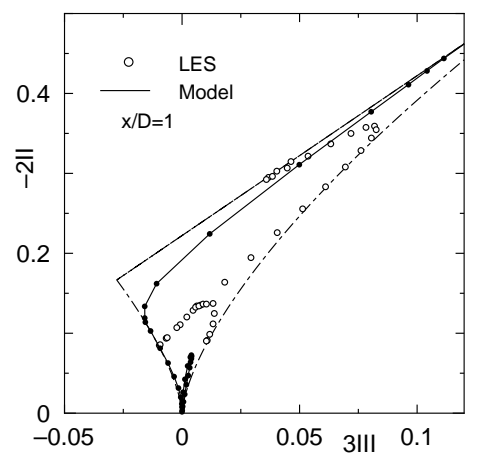

(n)

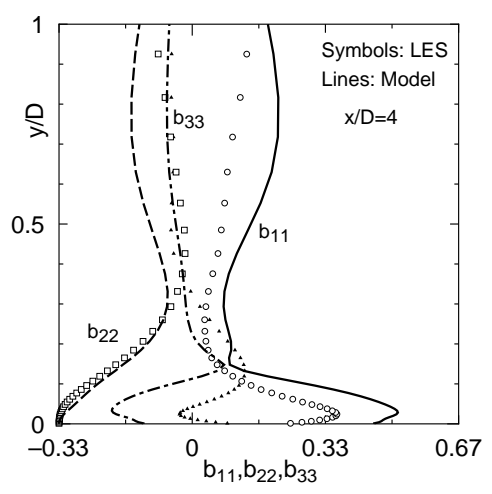

(i)

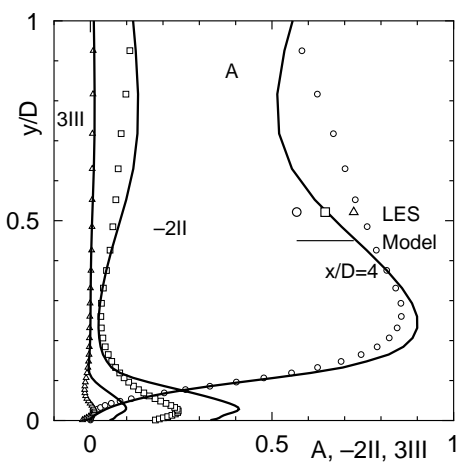

(1)

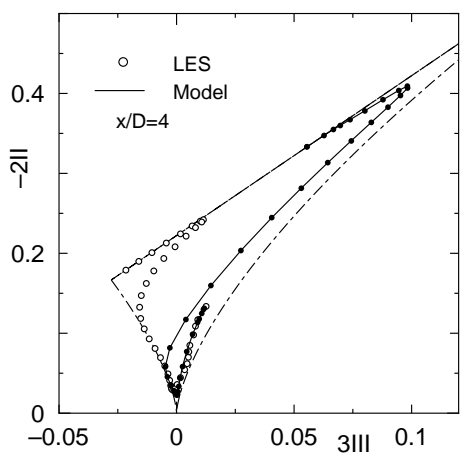

(o)

Figure 12: (continued)

«Abe et al.» 


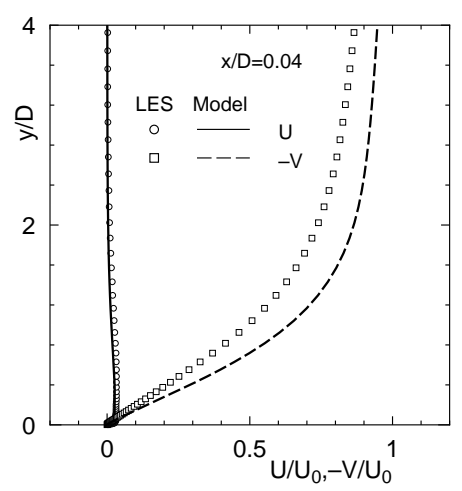

(a)

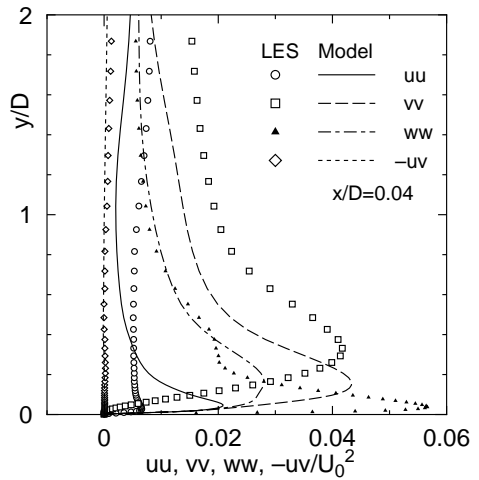

(d)

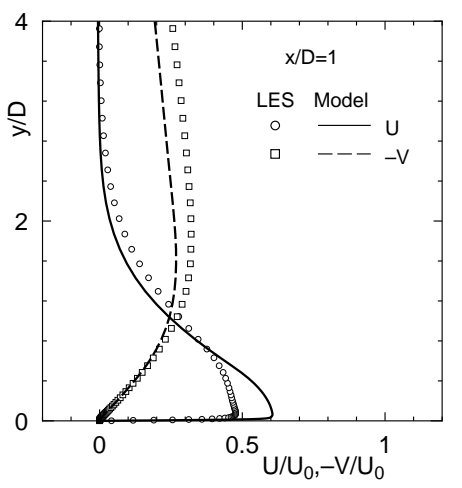

(b)

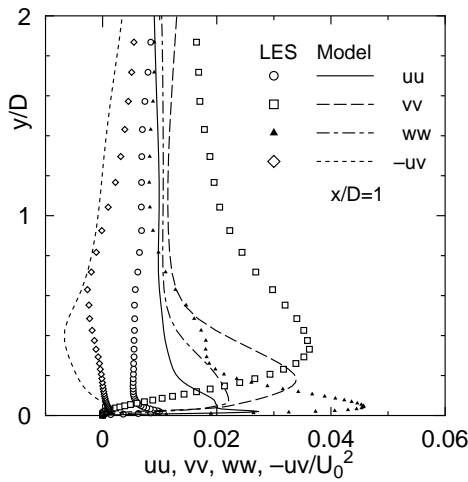

(e)

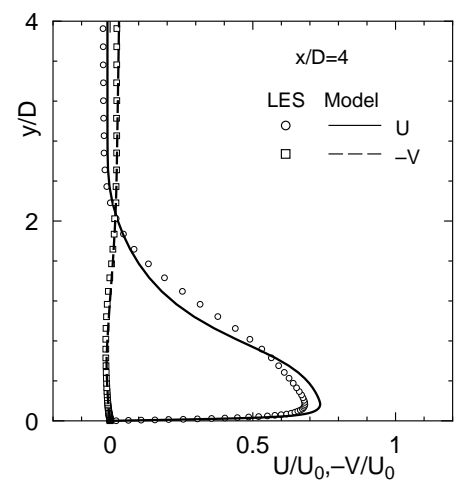

(c)

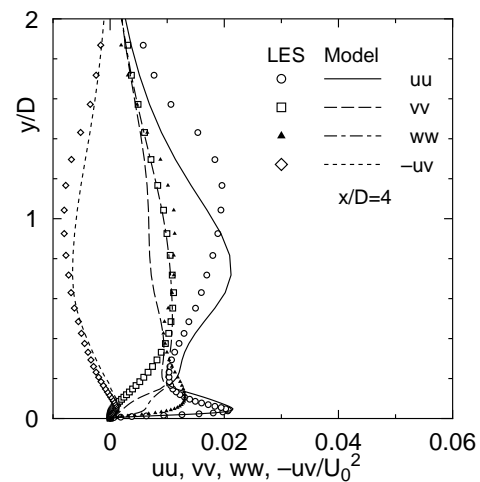

(f)

Figure 13:

$\ll$ Abe et al.» 


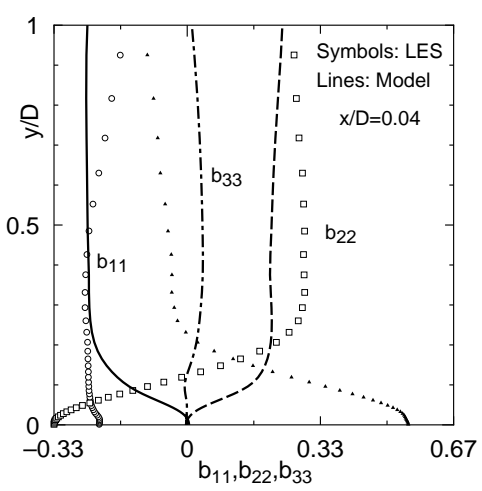

(g)

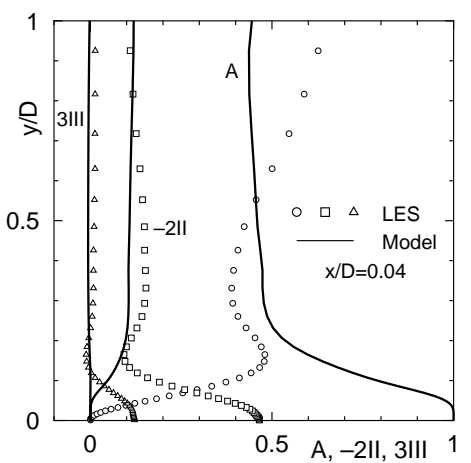

(j)

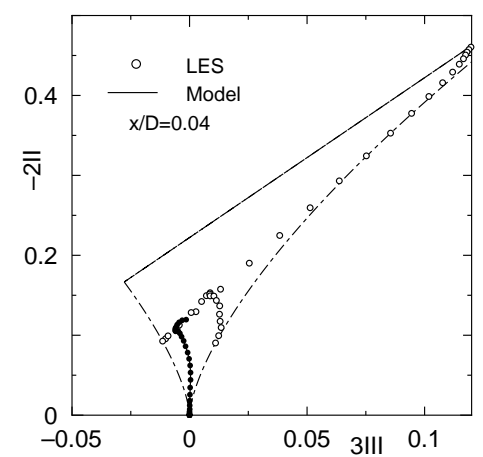

(m)

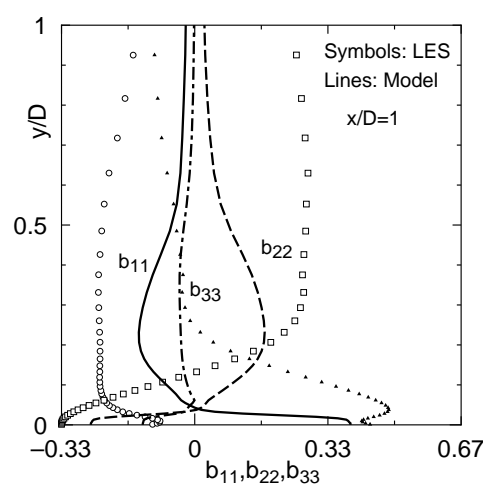

(h)

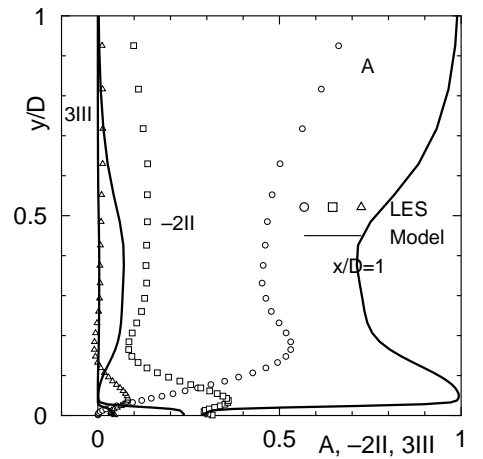

(k)

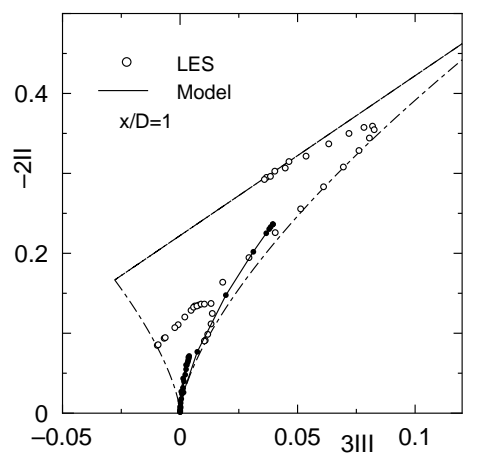

(n)

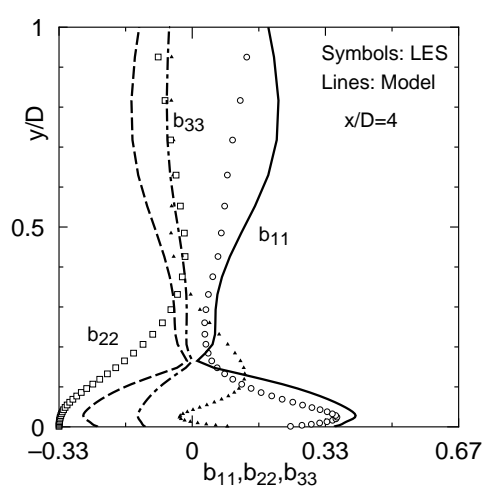

(i)

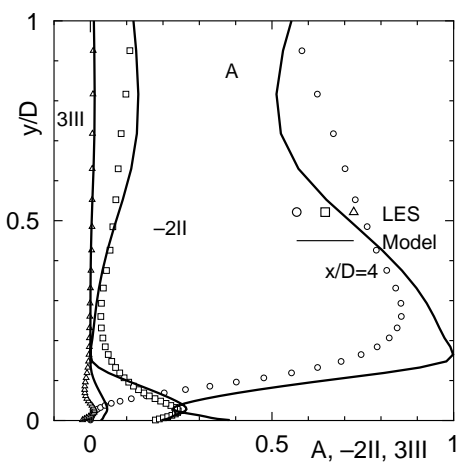

(l)

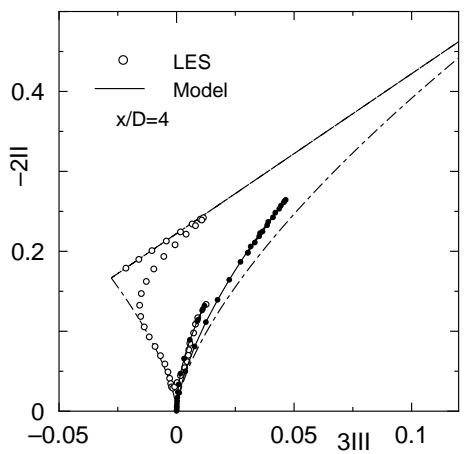

(o)

Figure 13: (continued)

«Abe et al.» 


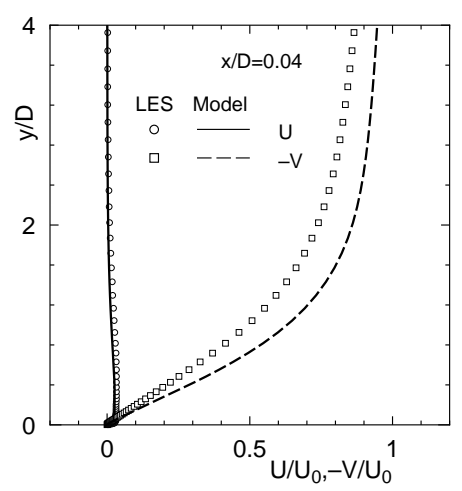

(a)

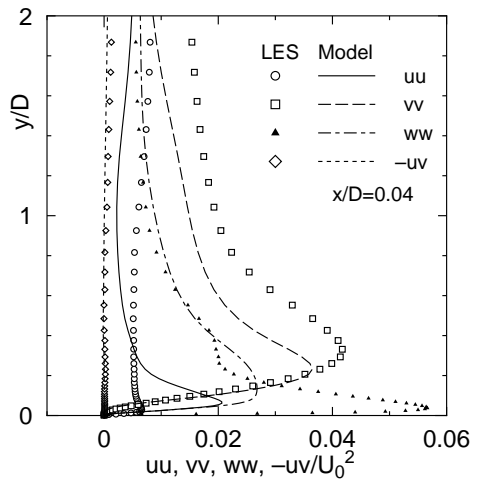

(d)

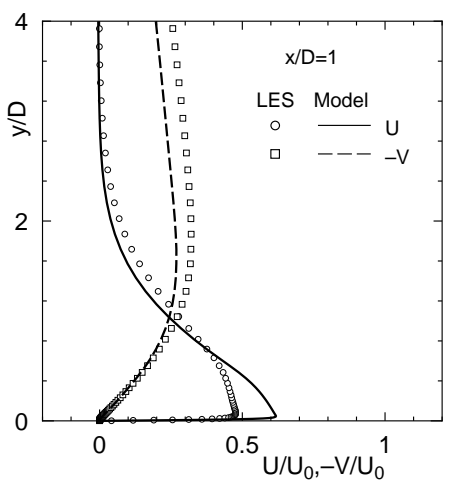

(b)

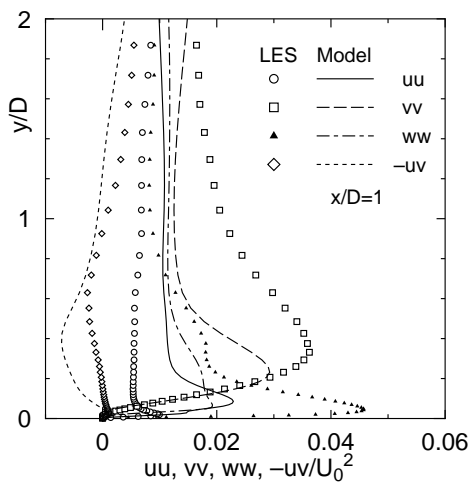

(e)

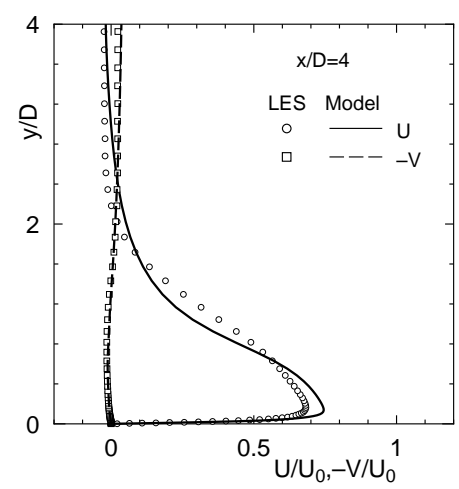

(c)

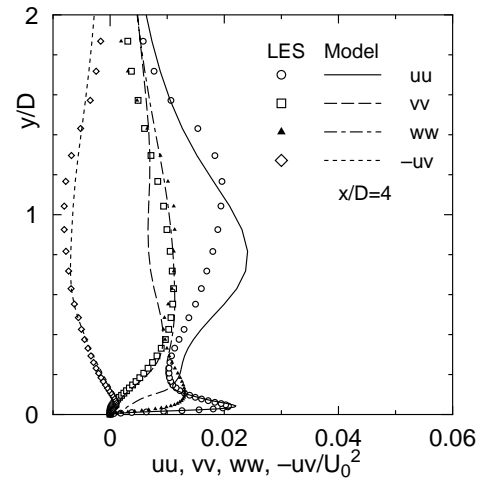

(f)

Figure 14:

$\ll$ Abe et al.» 


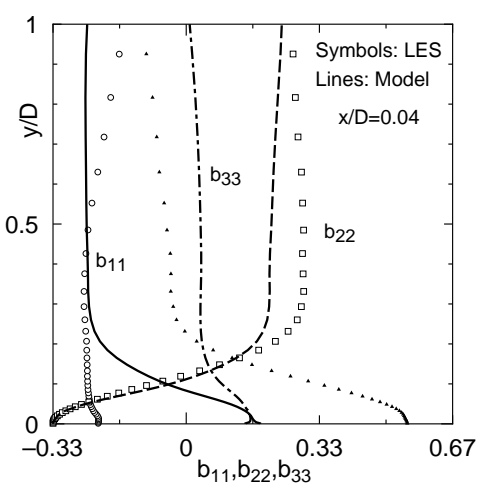

(g)

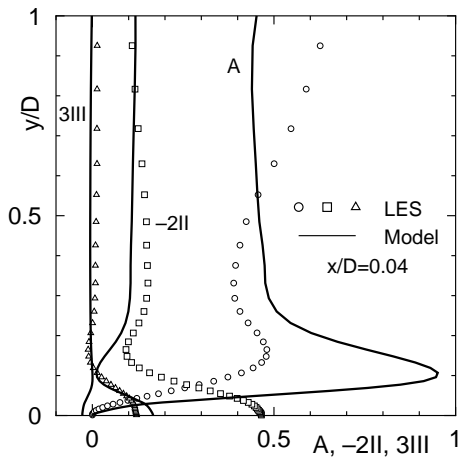

(j)

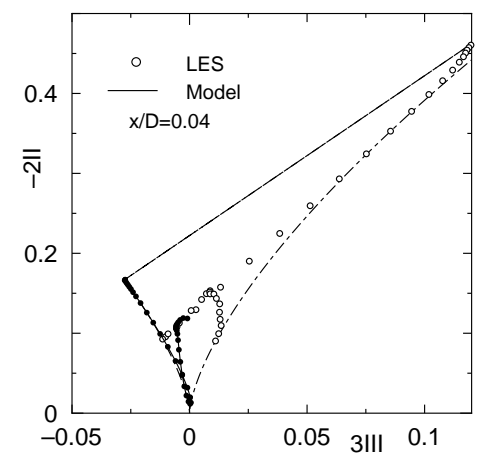

(m)

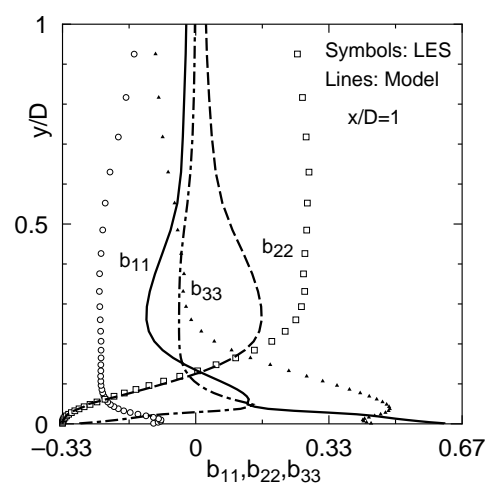

(h)

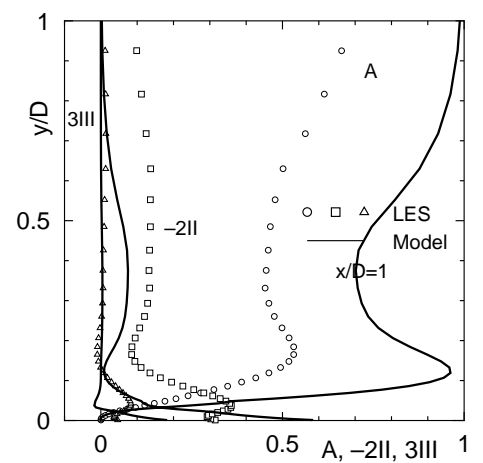

(k)

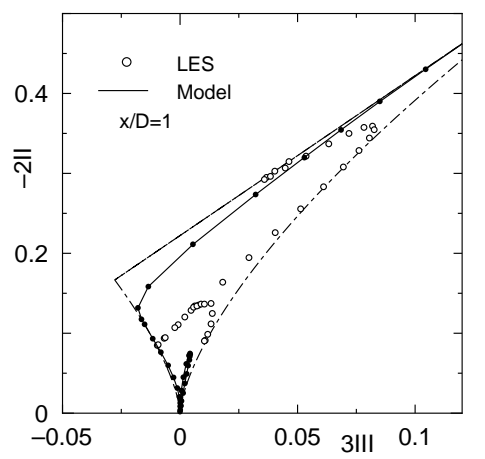

(n)

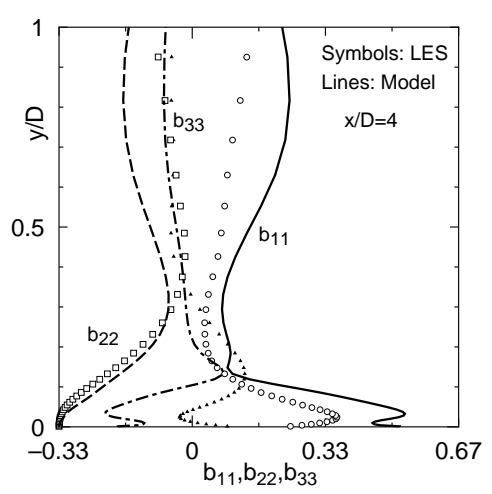

(i)

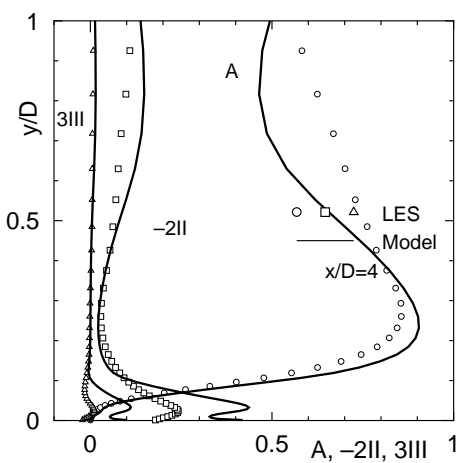

(1)

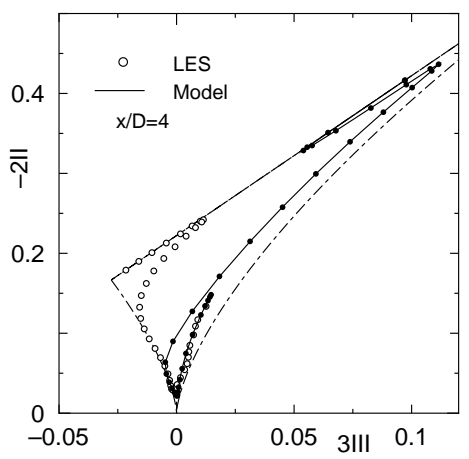

(o)

Figure 14: (continued)

«Abe et al.» 


\begin{tabular}{|c|c|}
\hline Figure 1: & $\ll$ Abe et al.» \\
\hline Figure 2: & $\ll$ Abe et al.» \\
\hline Figure 3: & $\ll$ Abe et al.» \\
\hline Figure 4: & $\ll$ Abe et al.» \\
\hline Figure 5: & $\ll$ Abe et al.» \\
\hline Figure 6: & $\ll$ Abe et al.» \\
\hline
\end{tabular}

Figure 7: $\quad \ll$ Abe et al.»

Figure 8: $\quad \ll$ Abe et al.»

Figure 9: $\quad \ll$ Abe et al.»

Figure 10: $\quad \ll$ Abe et al.»

Figure 11: $\quad$ «Abe et al.»

Figure 12: $\quad$ «Abe et al.»

Figure 13: $\quad$ «Abe et al.»

Figure 14: $\quad \ll$ Abe et al.» 